\title{
Chitosan-miRNA functionalized microporous titanium oxide surfaces via a layer-by-layer approach with a sustained release profile for enhanced osteogenic activity
}

Kaimin $\mathrm{Wu}^{1 \dagger}{ }^{1 \dagger}$, Mengyuan $\mathrm{Liu}^{2 \dagger}$, Nan $\mathrm{Li}^{3 \dagger}$, Li Zhang ${ }^{4}$, Fanhui Meng ${ }^{4}$, Lingzhou Zhao ${ }^{5 *}$, Min Liu ${ }^{1 *}$ and Yumei Zhang ${ }^{4^{*}}$

\begin{abstract}
Background: The biofunctionalization of titanium implants for high osteogenic ability is a promising approach for the development of advanced implants to promote osseointegration, especially in compromised bone conditions. In this study, polyelectrolyte multilayers (PEMs) were fabricated using the layer-by-layer approach with a chitosan-miRNA (CS-miRNA) complex and sodium hyaluronate (HA) as the positively and negatively charged polyelectrolytes on microarc-oxidized (MAO) Ti surfaces via silane-glutaraldehyde coupling.

Methods: Dynamic contact angle and scanning electron microscopy measurements were conducted to monitor the layer accumulation. RiboGreen was used to quantify the miRNA loading and release profile in phosphate-buffered saline. The in vitro transfection efficiency and the cytotoxicity were investigated after seeding mesenchymal stem cells (MSCs) on the CS-antimiR-138/HA PEM-functionalized microporous Ti surface. The in vitro osteogenic differentiation of the MSCs and the in vivo osseointegration were also evaluated.

Results: The surface wettability alternately changed during the formation of PEMs. The CS-miRNA nanoparticles were distributed evenly across the MAO surface. The miRNA loading increased with increasing bilayer number. More importantly, a sustained miRNA release was obtained over a timeframe of approximately 2 weeks. In vitro transfection revealed that the CS-antimiR-138 nanoparticles were taken up efficiently by the cells and caused significant
\end{abstract}

\footnotetext{
*Correspondence: zhaolingzhou1983@hotmail.com; dentist401@163.com; wqtzym@fmmu.edu.cn

†Kaimin Wu, Mengyuan Liu and Nan Li contribute equally to this work

${ }^{1}$ Department of Stomatology, Navy 971st Hospital, No. 22 Minjiang Road, Qingdao 266071, China

${ }^{4}$ State Key Laboratory of Military Stomatology \& National Clinical Research Center for Oral Diseases \& Shaanxi Key Laboratory of Stomatology, Department of Prosthodontics, School of Stomatology, The Fourth Military Medical University, No. 145 West Changle Road, Xi'an 710032, China

${ }^{5}$ State Key Laboratory of Military Stomatology \& National Clinical Research Center for Oral Diseases \& Shaanxi Engineering Research Center for Dental Materials and Advanced Manufacture, Department of Periodontology and Oral Medicine, School of Stomatology, The Fourth Military Medical University, No. 145 West Changle Road, Xi'an 710032, China

Full list of author information is available at the end of the article
}

(c) The Author(s) 2020. This article is licensed under a Creative Commons Attribution 4.0 International License, which permits use, sharing, adaptation, distribution and reproduction in any medium or format, as long as you give appropriate credit to the original author(s) and the source, provide a link to the Creative Commons licence, and indicate if changes were made The images or other third party material in this article are included in the article's Creative Commons licence, unless indicated otherwise in a credit line to the material. If material is not included in the article's Creative Commons licence and your intended use is not permitted by statutory regulation or exceeds the permitted use, you will need to obtain permission directly from the copyright holder. To view a copy of this licence, visit http://creativeco mmons.org/licenses/by/4.0/. The Creative Commons Public Domain Dedication waiver (http://creativecommons.org/publicdomain/ zero/1.0/) applies to the data made available in this article, unless otherwise stated in a credit line to the data. 
knockdown of miR-138 without showing significant cytotoxicity. The CS-antimiR-138/HA PEM surface enhanced the osteogenic differentiation of MSCs in terms of enhanced alkaline phosphatase, collagen production and extracellular matrix mineralization. Substantially enhanced in vivo osseointegration was observed in the rat model.

Conclusions: The findings demonstrated that the novel CS-antimiR-138/HA PEM-functionalized microporous Ti implant exhibited sustained release of CS-antimiR-138, and notably enhanced the in vitro osteogenic differentiation of MSCs and in vivo osseointegration. This novel miRNA-functionalized Ti implant may be used in the clinical setting to allow for more effective and robust osseointegration.

Keywords: microRNAs, Sustained release, Layer-by-layer, Microarc oxidation, Mesenchymal stem cells, Titanium implants

\section{Introduction}

Titanium (Ti) implants are widely applied as orthopaedic and dental implants. Although these implants have been successfully employed in the past, further development is required to meet the clinical needs for faster and tighter osseointegration [1, 2]. Conventional implant modification techniques, such as alteration of surface properties (e.g., suitable micro-, submicro- and nanoscale topographies and wettability [3-6]) and loading and delivery of inorganic elements $[7,8]$ are suggested to have limited effects on osteogenic activity enhancement [9]. Biofunctionalization of $\mathrm{Ti}$ implants with potent biochemical cues, such as extracellular matrix (ECM) proteins [10], peptides $[11,12]$, growth factors $[13,14]$ and nucleotides $[15,16]$, may result in more predictable osseointegration, especially in compromised bone conditions.

MicroRNAs (miRNAs) are endogenous small noncoding RNAs 19-25 nucleotides in length that play an important role in a variety of diverse and fundamental biological processes, such as cell development, proliferation, differentiation, apoptosis and signal transduction. It is believed that miRNAs mimic the natural differentiation pathway and delicately control multiple genes, constituting a much healthier stimulator of stem cell differentiation [17]. Recently, reports on the regulatory effect of miRNAs on osteogenesis have continued to expand [1821], among which miR-138 is reported to be a negative regulator of the osteogenic differentiation of mesenchymal stem cells (MSCs). AntimiR-138 inhibition of endogenous miR-138 levels was reported to enhance bone formation in vivo [18]. In our previous proof-of-concept studies, antimiR-138 lipoplexes were introduced to either the cell culture plate or the Ti surface to enhance the osteogenic differentiation of the MSCs cultured on these surfaces [22]. Before possible clinical application, it is important to use a more biocompatible carrier instead of liposomes and to control the loading and delivery kinetics of antimiR-138 to achieve a sustainable effect.

As a natural degradable cationic polymer, chitosan (CS), with good stability and cytocompatibility, could enhance drug internalization [23, 24] and has already been used for the delivery of DNA and RNA [25-28]. Via electrostatic interactions, CS can bind miRNAs to form relatively stable CS-miRNA nanoplexes (NPs) with a positive charge. Positively and negatively charged polyelectrolytes can be alternately adsorbed via electrostatic adsorption to fabricate a polyelectrolyte multilayer (PEM) film; this is referred to as layer-by-layer (LbL) technology $[29,30]$. PEMs with controlled loading and release profiles has been experimentally used to deliver growth factors [31, 32], DNA molecules [33, 34], RNA molecules [35, 36], etc.

It would be of special interest to use CS-antimiR-138 NPs and hyaluronic acid (HA, a commonly used polyanion in LbL) to PEM-functionalize Ti bone implants for sustainable antimiR-138 delivery and consequently augmented osseointegration. In addition, a more stable immobilization of the first layer is expected from chemical pretreatment of $\mathrm{Ti}$ with (3-aminopropyl) triethoxy silane (APTES) and glutaraldehyde (GA).

In this study, we developed a novel CS-antimiR-138/ HA PEM-functionalized microarc-oxidized (MAO) Ti implant by LbL and silane-glutaraldehyde coupling (Scheme 1). Following ultraviolet (UV) irradiation, a high number of surface hydroxyl groups was achieved by the facile and economical MAO treatment of $\mathrm{Ti}$ with high loading capacity [37]. The CS amine groups can form covalent bonds with the hydroxyl groups via silane-glutaraldehyde coupling of APTES and GA [38-40]. Furthermore, the osteogenic antimiR-138 was chosen for the PEM functionalization of the MAO Ti surface through LbL, and sustainable antimiR-138 delivery and enhanced osteogenic activity in vitro and in vivo was expected. We hope this work will provide beneficial information for loading miRNAs into other biomaterials.

\section{Materials and methods \\ Materials}

Commercially pure $\mathrm{Ti}$ discs $(15 \mathrm{~mm}$ in diameter and $2 \mathrm{~mm}$ in thickness) and screw Ti rods $(2.8 \mathrm{~mm}$ in diameter and $6 \mathrm{~mm}$ in length) were provided by the Northwest Institute for Nonferrous Metal Research, China. 
CS (molecular weight $100 \mathrm{kDa}$ and deacetylation degree of 95\%) was purchased from Jinke Co. Ltd (China). $\beta$-glycerophosphate disodium salt pentahydrate, calcium acetate monohydrate, APTES, GA, ascorbic acid, dexamethasone, Sirius Red, Alizarin Red and dimethyl sulfoxide (DMSO) were purchased from Sigma-Aldrich Company (USA). Solution RNase Erasol was bought from TIANDZ (China). $\alpha$-minimal essential medium $(\alpha-M E M)$, fetal bovine serum (FBS), penicillin and streptomycin were obtained from Hyclone (USA). Cell count kit-8 (CCK-8), lactate dehydrogenase (LDH), 3,39-dioctadecyloxacarbocyanine perchlorate (DiO) and BCIP/ NBT Alkaline Phosphatase Color Development Kit were provided by Beyotime (China). Phosphate buffered saline (PBS), 4',6-diamidino-2-phenylindole (DAPI) were purchased from Invitrogen (USA). E.Z.N.A. ${ }^{\mathrm{TM}}$ Total RNA Kit I was obtained from OMEGA (USA). PrimeScriptTM RT reagent kit and SYBR Premix ExTM Taq II were purchased from TaKaRa (Japan). Male SD rats (2 weeks old) were obtained from the laboratory animal center of the Fourth Military Medical University. The antimiR-138 (CGGCCUGAUUCACAACACCAGCU) and negative control (CAGUACUUUUGUGUAGUACAA) were synthesized from Gene Pharma Co., Ltd (China).

\section{Fabrication of CS-antimiR-138/HA PEM-functionalized microporous Ti through LbL}

Pure Ti discs were polished with 400- to 1500-grit waterproof abrasive paper and then ultrasonically cleaned in acetone, ethanol and distilled water for $10 \mathrm{~min}$ each. The $\mathrm{Ti}$ discs were then treated in an electrolyte containing $\beta$-glycerophosphate disodium salt pentahydrate $(0.02 \mathrm{M})$ and calcium acetate monohydrate $(0.2 \mathrm{M})$ at $400 \mathrm{~V} \mathrm{DC}$ for $5 \mathrm{~min}$. After ultrasonic cleaning and drying, the fabricated MAO Ti samples were sterilized for $30 \mathrm{~min}$ by UV irradiation.
$\mathrm{CS}$ and HA were dissolved in $0.2 \mathrm{M}$ sodium acetate buffer $(\mathrm{pH}=5.5)$ and deionized water $(\mathrm{pH}=5.5)$, respectively, at a concentration of $1 \mathrm{mg} / \mathrm{ml}$. Both CS and HA were sterilized using a $0.22 \mu \mathrm{m}$ syringe filter and then treated with the Solution RNase Erasol Kit according to the manufacturer's instructions. Afterwards, $100 \mu \mathrm{l}$ antimiR-138 (20 mM in RNA-free water) was added to $1 \mathrm{ml}$ final CS solution, quickly followed by magnetic stirring for $10 \mathrm{~min}$ and incubation for $30 \mathrm{~min}$ at room temperature. The N/P ratio (the molar ratio of chitosan amino groups to RNA phosphate groups) in our experiment was 60 , calculated with a mass per phosphate of $325 \mathrm{Da}$ for RNA and mass per charge of $163 \mathrm{Da}$ for chitosan (95\% deacetylation).

The CS-antimiR-138/HA PEM coating was then fabricated on MAO Ti surfaces via LbL. The dried MAO Ti discs were immersed in a $10 \%(\mathrm{v} / \mathrm{v})$ solution of APTES in alcohol in sealed individual containers for $2 \mathrm{~h}$ on a shaker. Then, the MAO discs were placed in pure alcohol and sonicated for $30 \mathrm{~min}$, which was repeated twice for a total sonication time of $90 \mathrm{~min}$. Afterwards, the $\mathrm{Ti}$ discs were rinsed with deionized water twice to remove residual alcohol and then dried. To link GA to the MAOAPTES surface, the GA solution $(2.5 \%(\mathrm{v} / \mathrm{v})$ in deionized water) was poured over the MAO-APTES samples ensuring complete coverage of the metal coupon. The containers were then sealed for $1 \mathrm{~h}$. Afterwards, the samples were rinsed thoroughly with deionized water 3 times. The CS dissolved in $0.2 \mathrm{M}$ sodium acetate buffer $(10 \mathrm{mg} /$ $\mathrm{ml}, \mathrm{pH}$ 5.5) was added to the Ti surface for $20 \mathrm{~min}$. After washing with sodium acetate buffer, the Ti sample was alternatively dipped into the HA solution and CS-antimiR-138 solution for 10 min each. Every dipping step was followed by two washing steps with sodium acetate buffer to remove excess materials. The steps were repeated until the desired number of bilayers was obtained.

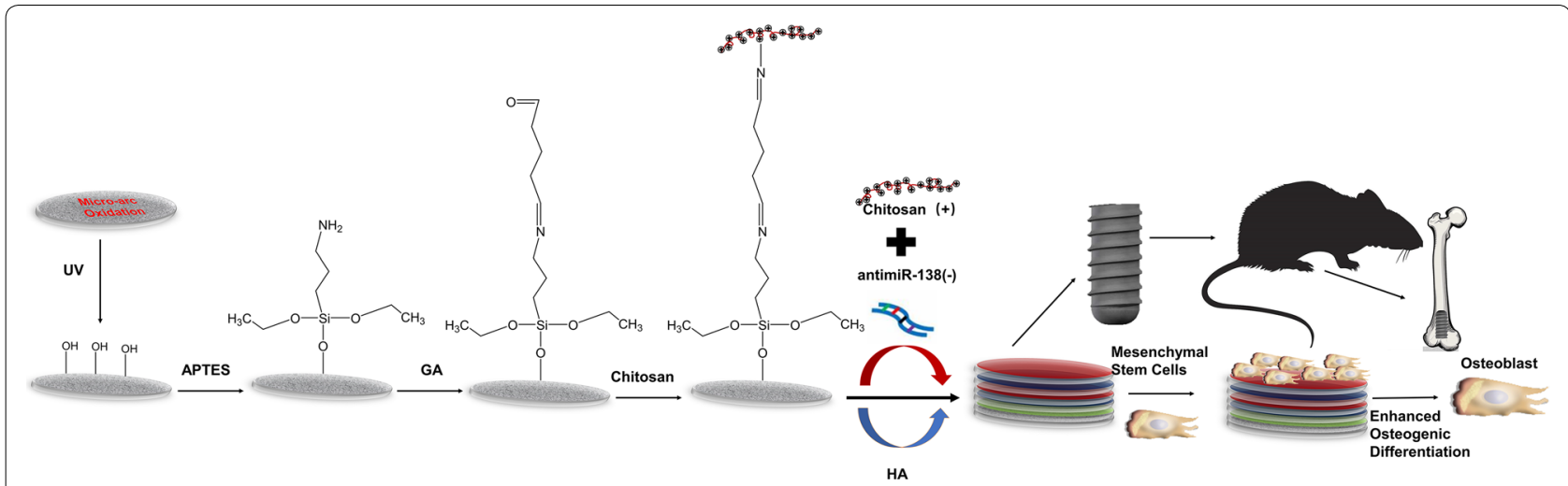

Scheme 1 Schematic diagram summarizing the fabrication of the CS-antimiR-138/HA PEM-functionalized microporous Ti implant through LbL with enhanced osteogenic activity 


\section{Characterization of the CS-antimiR-138/HA PEM-functionalized microporous $\mathrm{Ti}$}

The surface morphology of the prepared $\mathrm{Ti}$ samples was observed by field-emission scanning electron microscopy (FE-SEM, Hitachi S-4800, Japan). To assess the miRNA loading, Cy3-labelled miRNAs (Gene Pharma, China) were used to fabricate the coating. The Ti samples were observed by a laser scanning confocal microscope with a layer by layer scanning approach with an interlayer thickness of $400 \mathrm{~nm}$ (Fluo View, Olympus FV1000, Japan) immediately after fabrication or after 7 days of incubation in $\alpha$-MEM supplemented with $10 \%$ FBS. The threedimensional images were reconstructed.

\section{Quantification of the loading and release of miRNA from CS-antimiR-138/HA PEM-functionalized microporous Ti}

To measure the miRNA loading within each layer of the functionalized surface, droplets of $40 \mu \mathrm{l} \mathrm{CS-miRNA}$ nanoparticles were dropped discreetly in the experiment and incubated for $10 \mathrm{~min}$. Then, the coated surface was washed twice with $40 \mu \mathrm{l}$ sodium acetate buffer. Afterwards, the washing solution was carefully collected and quantitatively analysed by the RiboGreen assay according to the manufacturer's instructions. Finally, the miRNA loading in each layer was calculated by subtracting the miRNA amount in the washing solution from the amount in the original $40 \mu \mathrm{l} \mathrm{CS-miRNA} \mathrm{nanoparticle} \mathrm{suspension.}$ The total miRNA loading in the PEMs was calculated as the sum of the miRNA amount in each layer.

The CS-antimiR-138/HA PEM-functionalized microporous $\mathrm{Ti}$ samples were incubated in $300 \mu \mathrm{l}$ PBS $(\mathrm{pH}=7.4)$ at $37{ }^{\circ} \mathrm{C}$ in $5 \% \mathrm{CO}_{2}$ and $100 \%$ humidity for 14 days. At 8, 16, and $24 \mathrm{~h}$ and $2,3,4,6,8,10,12$ and 14 days, the extracting solution was replaced with $300 \mu \mathrm{l}$ of fresh PBS solution. The released miRNA in the collected PBS solution was quantified with the RiboGreen assay. As a reference, a standard curve of the miRNA concentration was determined with multiple dilutions of miRNA in PBS and PBS alone.

\section{Cell culture}

Primary rat bone marrow MSCs were obtained from 2 -week-old Sprague-Dawley rats. The cells were cultured in $\alpha$-MEM supplemented with $10 \%$ FBS and $1 \%$ penicillin/streptomycin and incubated in a humidified atmosphere of $5 \% \mathrm{CO}_{2}$ at $37{ }^{\circ} \mathrm{C}$. Passages 2-4 were used in the experiments. The MAO surface and tissue culture plate served as controls. The medium was replaced twice every week.

\section{Transfection efficiency assay}

Functionalized Ti samples made of Cy3-labelled miRNAs were used for this assay. MSCs of $2.5 \times 10^{4} / \mathrm{cm}^{2}$ were inoculated on $\mathrm{Ti}$ samples placed in 24-well plates. After $48 \mathrm{~h}$ of culture, the transfected cells were harvested by treatment with trypsin, washed with PBS and fixed in $1 \%$ paraformaldehyde. To observe the internalization of the Cy3-labelled miRNAs into the cells, the cells were fixed with $4 \%$ paraformaldehyde and washed with PBS. The cell membrane was stained with $\mathrm{DiO}$. The cell nucleus was stained with DAPI. The DiO, DAPI and Cy3 fluorescence signals were then observed by laser scanning confocal microscopy. To measure the miR-138 amount in the cells, the RNA in the cells was collected and reversetranscribed using a PrimeScript RT reagent kit and a specific reverse transcription primer (Gene Pharma, China) according to the manufacturer's recommendation. The miR-138 amount was quantified using a real-time polymerase chain reaction (real-time PCR) system (CFX96 ${ }^{\mathrm{TM}}$, Bio-Rad, USA) with SYBR Premix Ex Taq ${ }^{\mathrm{TM}}$ II. U6 small nuclear RNA was used as an endogenous normalization control.

\section{Cell morphology}

MSCs were seeded at a density of $5 \times 10^{4}$ cells/well. After $24 \mathrm{~h}$ of culture, the Ti samples with attached cells were gently washed with PBS, fixed in $2.5 \%$ glutaraldehyde, dehydrated in a graded ethanol series and freeze-dried. After sputter coating with carbon, the cell morphology was observed by FE-SEM.

\section{In vitro osteogenesis of MSCs}

The expression of osteogenisis-related genes was evaluated using the real-time PCR. The cells were seeded with $5 \times 10^{4}$ cells/well and cultured for $48 \mathrm{~h}$. Then the medium was changed into osteogenic medium containing $10 \mathrm{mM} \beta$-glycerophosphate, $50 \mu \mathrm{g} / \mathrm{ml}$ ascorbic acid and $10^{-7} \mathrm{M}$ dexamethasone. After further culture of 7 and 14 days, the total RNA was isolated using the TRIzol reagent. Then $2 \mu \mathrm{g}$ RNA from each sample was reversed transcribed into complementary DNA (cDNA) using the PrimeScript RT reagent kit. The expression of osteogenesis-related genes including collagen type I (COL1), runt-related transcription factor 2 (RUNX2), alkaline phosphatase (ALP), bone morphogenetic protein-2 (BMP-2), osterix (OSX) and osteocalcin (OCN) was quantified using real-time PCR. The PCR reaction was carried out using SYBR Premix Ex Taq ${ }^{\mathrm{TM}}$ II on the $\mathrm{CFX}^{\mathrm{TM}}{ }^{\mathrm{TM}}$ real-time PCR System. The relative expression levels for each gene of interest were normalized to that of the housekeeping gene GAPDH. The PCR primers were synthesized as shown in Table 1. 


\section{ALP staining}

The cell inoculation and culture was the same as in the real-time PCR assay. After culturing for 7 and 14 days, the cells on the Ti samples were washed with PBS and fixed with $4 \%$ paraformaldehyde. ALP was stained with the BCIP/NBT ALP color development kit for $15 \mathrm{~min}$. The samples were washed thoroughly with PBS to acquire the images.

\section{Collagen secretion}

The cell inoculation and culture was the same as in the real-time PCR assay. After culture of 7 and 14 days, the cultures were washed by PBS and fixed by $4 \%$ paraformaldehyde. Then the collagen secretion was stained by $0.1 \mathrm{wt} \%$ Sirius red in saturated picric acid for $18 \mathrm{~h}$. The unbound stain was removed in $0.1 \mathrm{M}$ acetic acid and then the images were collected. To quantitatively assess the collagen secretion, the stain on the samples was eluted in $500 \mu \mathrm{l}$ destain solution $(0.2 \mathrm{M} \mathrm{NaOH} /$ methanol 1:1) and the optical density at $540 \mathrm{~nm}$ was measured using a spectrophotometer.

\section{ECM mineralized nodule displaying}

The cell inoculation and culture was the same as in the real-time PCR assay. After culturing for 14 and 28 days, the cells were washed twice with PBS and then fixed with $60 \%$ isopropanol for $1 \mathrm{~min}$. After rehydrating with distilled water for 2-3 min, the ECM mineralized nodules formed by MSC culture were stained with $1 \mathrm{wt} \%$ alizarin red for $3 \mathrm{~min}$. After thorough washing with distilled water, the images were taken.

\section{In vivo osseointegration}

\section{Implant surgery}

The animal experiment was approved by the Animal Research Committee of the Fourth Military Medical University and conducted in accordance with the international standards on animal welfare. Twenty female Sprague-Dawley rats, 3 months old with a weight of approximately $250 \mathrm{~g}$, were randomly divided into 5 groups: antimiR-138 group, antimiR-control group, CS group, MAO group and polished Ti (PT) group $(\mathrm{n}=8$ for each group). The femur model was applied for implantation in this study, and the procedure of the surgery was similar to the literature procedure [41]. Briefly, after anaesthesia by pelltobarbitalum natricum, the hind limbs were prepared by shaving and cleaning with ethanol and $10 \%$ povidone iodine. Then, an incision was made over the distal side of the knee. Gentle dissection was used to move the ligament and patella aside to expose the intercondylar notch of the distal femur. For placement of the screw implant, dental burs and a surgical motor (OsseoSet 200, Nobel Biocare AB, Sweden) with a low rotational drill speed $(800 \mathrm{rpm})$ were used to generate a $2.8 \mathrm{~mm}$ cylindrical hole at the intercondylar notch of the femur parallel to the long axis of the bone cooled continuously with sterile saline solution. Implants were inserted into the femoral medullary canal, followed by incision suture. The animals received an injection of gentamicin $(1 \mathrm{mg} /$ $\mathrm{kg}$ ) immediately after surgery and for 5 days after the surgery.

\section{Micro-CT evaluation}

Specimens (femurs containing implants) were extracted ( $\mathrm{n}=4$ per group) and fixed in $4 \%$ paraformaldehyde for $24 \mathrm{~h}$. Then, they were scanned by a micro-CT scanner (Y.Cheetah, YXLON International GmbH, Germany). The region of interest (ROI) was defined as the region at $2 \mathrm{~mm}$ height from $0.5 \mu \mathrm{m}$ below the growth plate and $200 \mu \mathrm{m}$ around the implant surface, and the images were analysed via VGStudio Max 2.2 (Volume Graphic, Germany). The bone volume per total volume (BV/TV), the mean trabecular thickness (Tb.Th), the mean trabecular number (Tb.N) and the mean trabecular separation ( $\mathrm{Tb}$. $\mathrm{Sp})$ were assessed within the ROI zone.

\section{Van Gieson staining}

After micro-CT scanning, the specimens were dehydrated with graded alcohol and embedded in methyl methacrylate. Afterwards, thin sections (approximately $50 \mu \mathrm{m}$ in thickness) parallel to the long axis of the implants were prepared using a macrocutting and

Table 1 Primers used for real-time PCR

\begin{tabular}{lll}
\hline Gene & Forward primer sequence $\mathbf{( 5}^{\prime} \mathbf{- 3}^{\prime} \mathbf{)}$ & Reverse primer sequence $\mathbf{5}^{\prime} \mathbf{- 3}^{\prime \mathbf{}} \mathbf{)}$ \\
\hline ALP & AACGTGGCCAAGAACATCATCA & TGTCCATCTCCAGCCGTGTC \\
BMP-2 & CAACACCGTGCTCAGCTTCC & TTCCCACTCATTTCTGAAAGTTCC \\
COL1 & GCCTCCCAGAACATCACCTA & GCAGGGACTTCTTGAGGTTG \\
OCN & GGTGCAGACCTAGCAGACACCA & AGGTAGCGCCGGAGTCTATTCA \\
RUNX2 & CCATAACGGTCTTCACAAATCCT & TCTGTCTGTGCCTTCTTGGTTC \\
OSX & AAGGCAGTTGGCAATAGTGG & TGAATGGGCTTCTTCCTCAG \\
GAPDH & GGCACAGTCAAGGCTGAGAATG & ATGGTGGTGAGACGCCAGTA \\
\hline
\end{tabular}


grinding system (SP1600 and SP2600, Leica, Germany). Sections were then polished and stained with $1.2 \%$ trinitrophenol and 1\% acid fuchsin (Van Gieson staining). Finally, a qualitative analysis of the bone formation was performed with a standard light microscope (M80, Leica, Germany) equipped with a digital image analysis system (Image-Pro Plus software, USA). The bone-to-implant contact (BIC) was also determined.

\section{Line-scanning of the bone-to-implant interface}

Line scanning by energy-dispersive X-ray spectroscopy (EDX, Hitachi, Japan) was applied for a more detailed analysis of the bone-to-implant interface. After having been fixed with $4 \%$ paraformaldehyde, dehydrated with graded alcohol, and embedded in methyl methacrylate, the bone-to-implant interfaces of the embedded samples were scanned by FE-SEM. The line profiles of $\mathrm{C}, \mathrm{O}, \mathrm{Ca}, \mathrm{P}$, and $\mathrm{Ti}$ elements were analysed from the implant side to the medullary cavity side by EDX.

\section{Statistical analysis}

The one-way ANOVA and Turkey post hoc tests were used to determine the level of statistical significance of difference among groups. $p<0.05,0.01$ and 0.001 was set to be significant, highly significant and extremely significant, respectively.

\section{Results}

Characterization of the CS-antimiR-138/HA

PEM-functionalized microporous Ti surface

\section{Contact angle measurement}

During the growth of the CS-antimiR-138/HA PEMfunctionalized microporous Ti surface, we monitored the wettability of each layer. As shown in Fig. 1, the naked MAO Ti surface (0 layer) was hydrophilic with the lowest water contact angle of $\sim 20^{\circ}$. The initially adsorbed APTES and GA layers, named layers 1 and 2, respectively, were both less hydrophilic than the naked MAO, showing contact angles of approximately $50^{\circ}$ and $35^{\circ}$. After the growth of layer 3 on the surface, which was CS, the contact angle decreased. The following layers displayed an alternating decrease or increase in their contact angles until layer 13. In general, the HA layer on the surface produced lower contact angles than the CS-antimiR-138 layer.

\section{SEM and fluorescent observation}

The morphology of the MAO Ti surfaces before and after PEM functionalization was observed by FE-SEM (Fig. 2). The naked MAO surface exhibited the typical MAO microporous structure (Fig. 2a-c). After the silaneglutaraldehyde coupling process, there was no apparent change in the structure (Fig. 2d-f). For the CS-miRNA/ HA PEM-functionalized microporous Ti surface, the CSmiRNA NPs were distributed evenly across the microporous structure (Fig. $2 \mathrm{~g}-\mathrm{i}$ ). The presence of the miRNA NPs slightly decreased the diameters of the micropores, and the NPs even completely filled some of the smaller micropores by attaching to their sidewalls.

To observe the miRNA loading and release, the Cy3-labelled miRNAs were used to fabricate the CSantimiR-138/HA PEM-functionalized microporous Ti surface. The surface was observed by laser scanning confocal microscopy from the surface to the bottom with a layer-by-layer scanning approach with an interlayer distance of $400 \mathrm{~nm}$ (Fig. 3a-i). From the

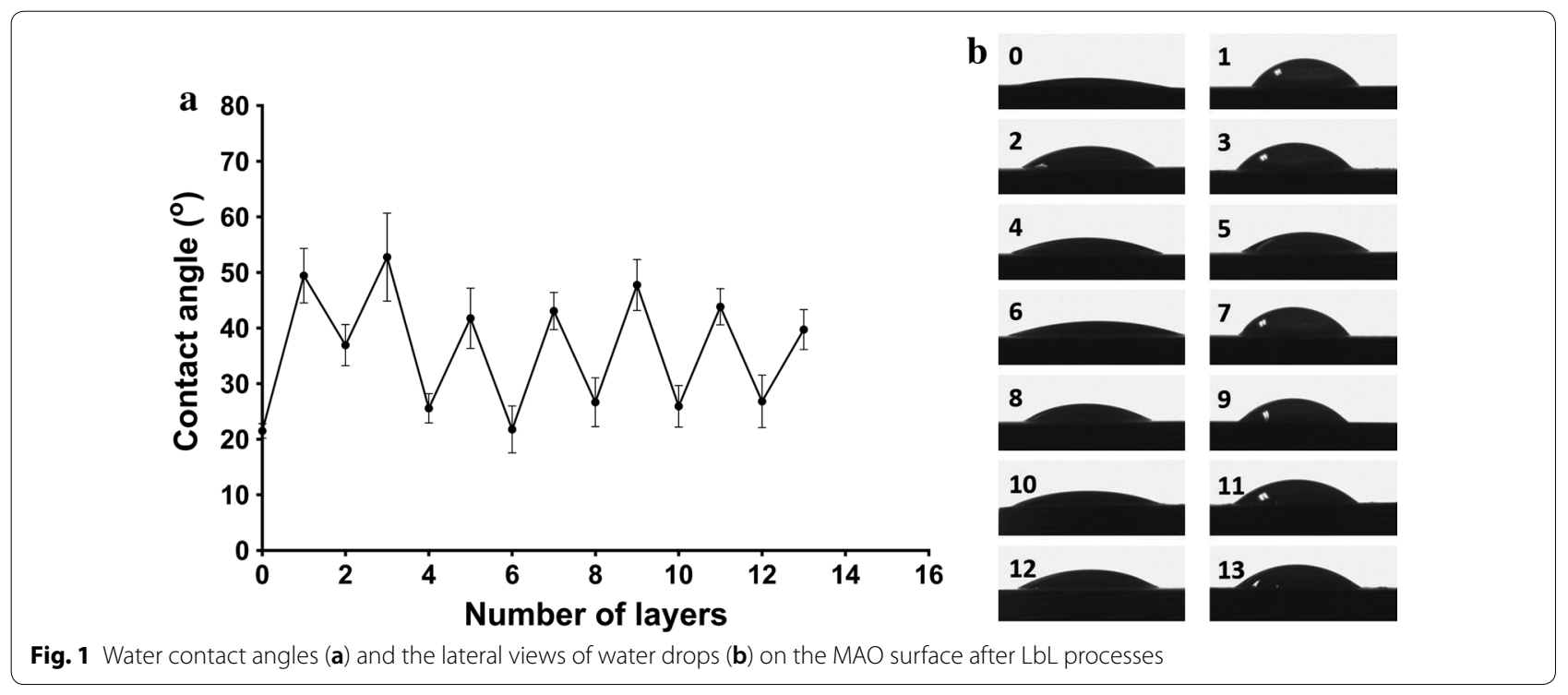


fluorescence images, the miRNAs were distributed relatively evenly and internalized into the pores. Figure $3 \mathrm{a}$ and $\mathrm{i}$ show the top and bottom layers of the CS-antimiR-138/HA PEM coating, respectively. The total thickness of the CS-antimiR-138/HA PEM coating was estimated to be approximately $3.2 \mu \mathrm{m}$. From the middle layers (Fig. 3b-h), we can observe many fluorescence rings attributed to the CS-miRNA NPs that attached to the sidewalls of the micropores. To display the miRNA release, the three-dimensional images (Fig. 3j, k) were reconstructed through the software after layer-by-layer scanning, and they show the miRNA amount before and after 7 days of immersion in the culture medium. A certain amount of miRNAs was retained on the surface and the sidewalls of the micropores after 7 days, indicating a high Ti surface retention of the CS-miRNA NPs and potential sustained release of the miRNAs from the surface.

\section{Quantification of miRNA loading and release of the CS-miRNA/HA PEM-functionalized microporous Ti surface}

The accumulative loading profiles were calculated (Fig. 4). The accumulated miRNA loading increased gradually with the layer number, indicating that the LbL approach can be used to control the miRNA loading (Fig. 4a). The miRNAs released steadily from the CSmiRNA/HA PEM-functionalized microporous Ti surface without apparent initial burst release (Fig. 4b). In the first 4 days, nearly $50 \%$ of the loaded miRNAs were released from the functionalized surface. After 14 days, a total of $\sim 0.8 \mu \mathrm{g}$ was released, which was nearly $100 \%$ of the loaded amount.
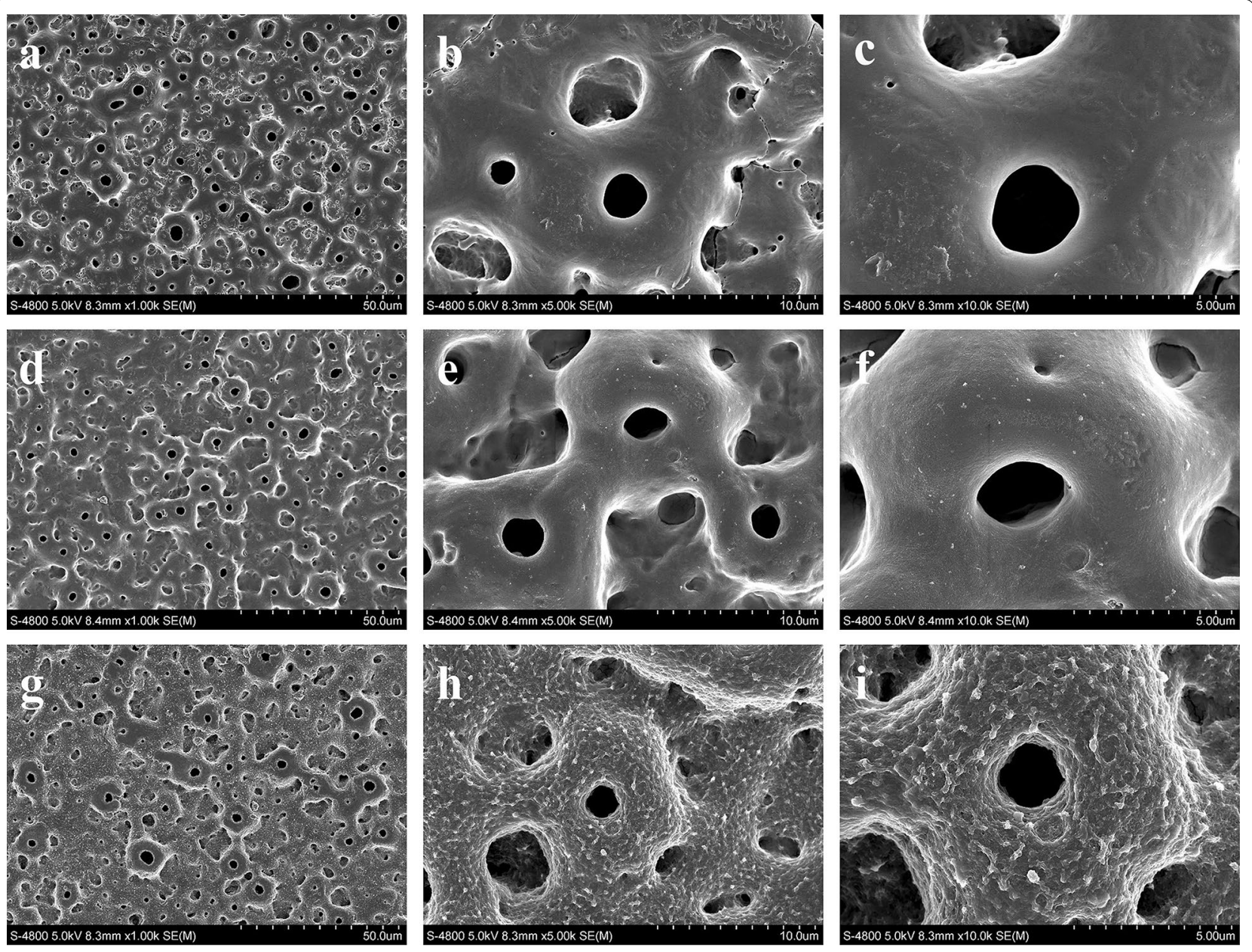

Fig. 2 Morphology of the MAO Ti surfaces before and after CS-miRNA/HA PEM-functionalized inspected by FE-SEM: (a, $\mathbf{b}$ and $\mathbf{c})$ pictures of different magnification for the naked MAO surface, (d, e and $\mathbf{f}$ ) pictures of silane glutaraldehyde functionalized MAO surface, ( $\mathbf{g}, \mathbf{h}$ and $\mathbf{i})$ pictures of 5 layers for the CS-miRNA/HA PEM-functionalized MAO surface 

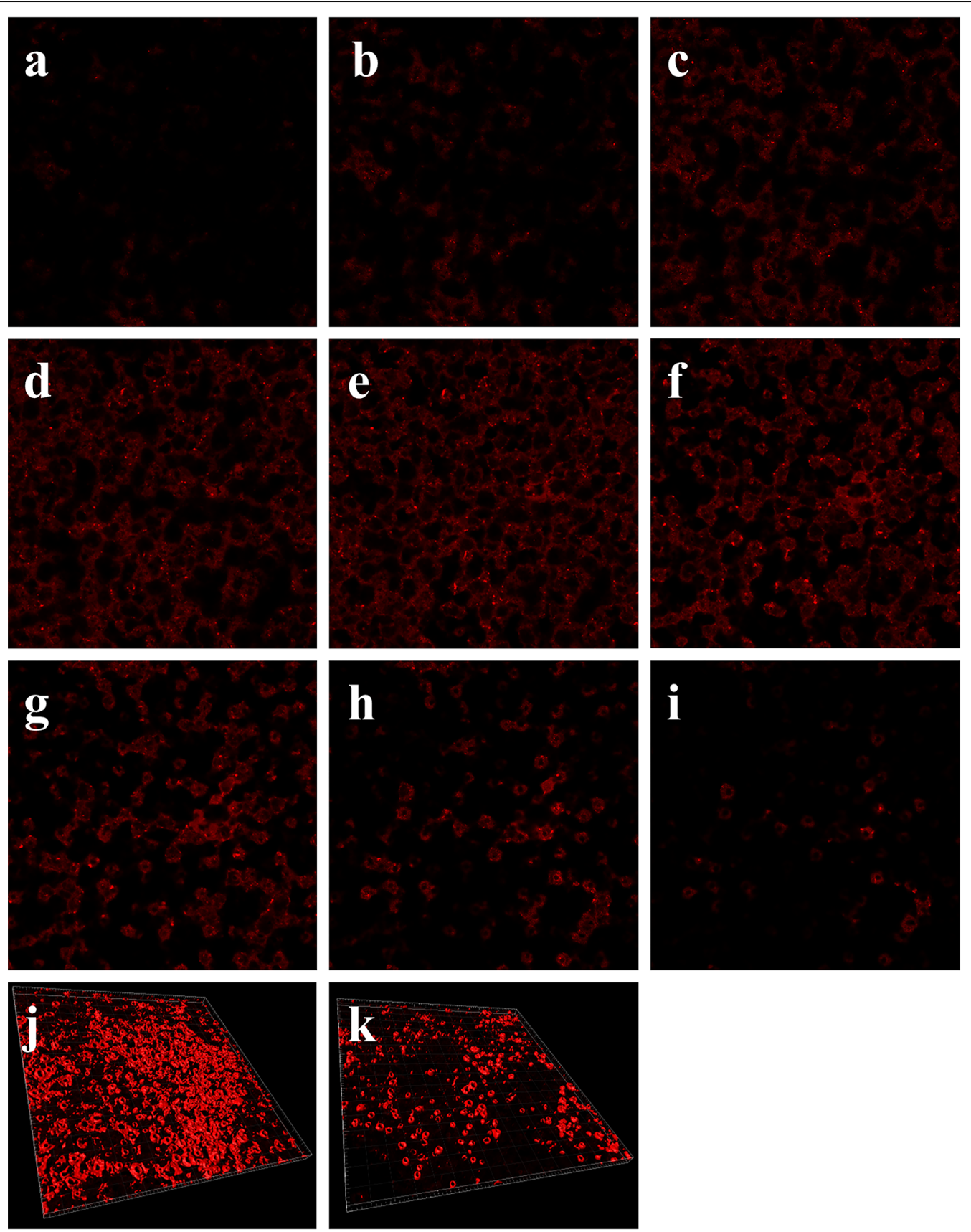

Fig. 3 Fluorescence confocal laser scanning microscope of CS-miRNA/HA PEM-functionalized MAO surface with Cy3-labeled miRNAs: a the top layer starting to display fluorescence and $\mathbf{b}-\mathbf{i}$ the continuing layers from top to down with an interlayer distance of $400 \mathrm{~nm}$. The fluorescence 3-D images of Cy3-labeled miRNAs on the MAO surfaces before $(\mathbf{j})$ and after $(\mathbf{k}) 7$ days of incubation in cell culture medium at $37^{\circ} \mathrm{C}$

\section{Transfection efficiency of the CS-miRNA/HA}

\section{PEM-functionalized microporous Ti surface}

To observe the internalization of the miRNAs into the cells, Cy3-labelled antimiR-138 was used to fabricate the functionalized Ti surface before seeding MSCs on the surface for $24 \mathrm{~h}$ of transfection. The miRNAs were red, and the cell nucleus and membrane were stained with blue and green colours, respectively (Fig. 5a). We observed that after $24 \mathrm{~h}$ of incubation, the miRNAs were mainly located in the cell body and around the nucleus, suggesting successful uptake of the CS-miRNA NPs by the cells. Most cells have intracellular miRNAs, indicating a high transfection efficiency of the functionalized $\mathrm{Ti}$ surface. 
To assess the downregulatory effect of the CS-antimiR-138/HA PEM-functionalized microporous Ti surface on the level of intracellular miR-138, the amount of intracellular miR-138 was measured by miRNA PCR (Fig. 5b). The miR-138 level in MSCs seeded on the CSantimiR-138/HA PEM-functionalized microporous $\mathrm{Ti}$ surface was successfully downregulated by approximately $70 \%$.

\section{Cell attachment and morphology}

The MSC attachment and cell spreading on the Ti samples were observed by FE-SEM (Fig. 6). Generally, the cells attached well to the microporous structure, and showed very similar polygonal cell morphology and a similar spreading area on the microporous $\mathrm{Ti}$ surface and the PEM-functionalized microporous $\mathrm{Ti}$ surfaces with antimiR-138 or antimiR-control. However, on the CS-antimiR-138/HA PEM-functionalized microporous Ti surface, the filopodia extended from the cells seemed to be thicker than those on the other two surfaces. The cellular filopodia on the CS-antimiR-138/HA PEMfunctionalized microporous Ti surface were anchored in the micropores. This phenomenon was not observed on the other two control surfaces. Furthermore, there were many granules on the dorsal side of the cells on the CSantimiR-138/HA PEM-functionalized microporous Ti surface, indicating that the cells on the CS-antimiR-138/ HA PEM-functionalized microporous Ti surface have a more mature secretory phenotype.

\section{Effect of the CS-antimiR-138/HA PEM-functionalized microporous Ti surface on osteogenesis in vitro}

The expression levels of osteogenesis-related genes were assessed by real-time PCR (Fig. 7). Generally, the CSantimiR-138/HA PEM-functionalized microporous Ti surface induced strikingly higher expression levels of BMP-2, OCN, COL1, ALP, OSX and RUNX2 by MSCs than the CS-antimiR-control/HA PEM-functionalized microporous Ti surface, the CS-coated surface and the naked MAO surface. There was no obvious difference in the expression levels of those genes among the CSantimiR-control/HA PEM-functionalized microporous Ti surface, the CS-coated surface or the naked MAO surface.

Then, the ALP production and collagen secretion after 7 days of culture as well as the ECM mineralization after 14 days of culture were assessed (Fig. 8). The MSCs produced the most ALP on the CS-antimiR-138/HA PEMfunctionalized microporous Ti surface after 7 days of osteogenic induction (Fig. 8a-d). There was no obvious difference in the induced ALP production levels among the antimiR-control/HA PEM-functionalized microporous Ti surface, the CS-coated surface or the naked MAO surface.

Collagen secretion was stained with Sirius red, and the results are shown in Fig. $8 a^{\prime}-d^{\prime}$. The optical images show that much more collagen was secreted by the cells on the CS-antimiR-138/HA PEM-functionalized microporous Ti surface compared to the collagen secretion on the antimiR-control/HA PEM-functionalized microporous Ti surface, the CS-coated surface and the naked MAO surface. The quantitative results indicate that the amount of secreted collagen on the CS-antimiR-138/HA PEMfunctionalized microporous Ti surface was approximately double that on the naked MAO surface and triple that on the antimiR-control/HA PEM-functionalized microporous Ti surface and the CS-coated surface.

Next, the ECM mineralized nodules were stained via alizarin red, and the results are shown in Fig. $8 a^{\prime \prime}-d^{\prime \prime}$. The mineralized nodules formed by the MSCs were observed
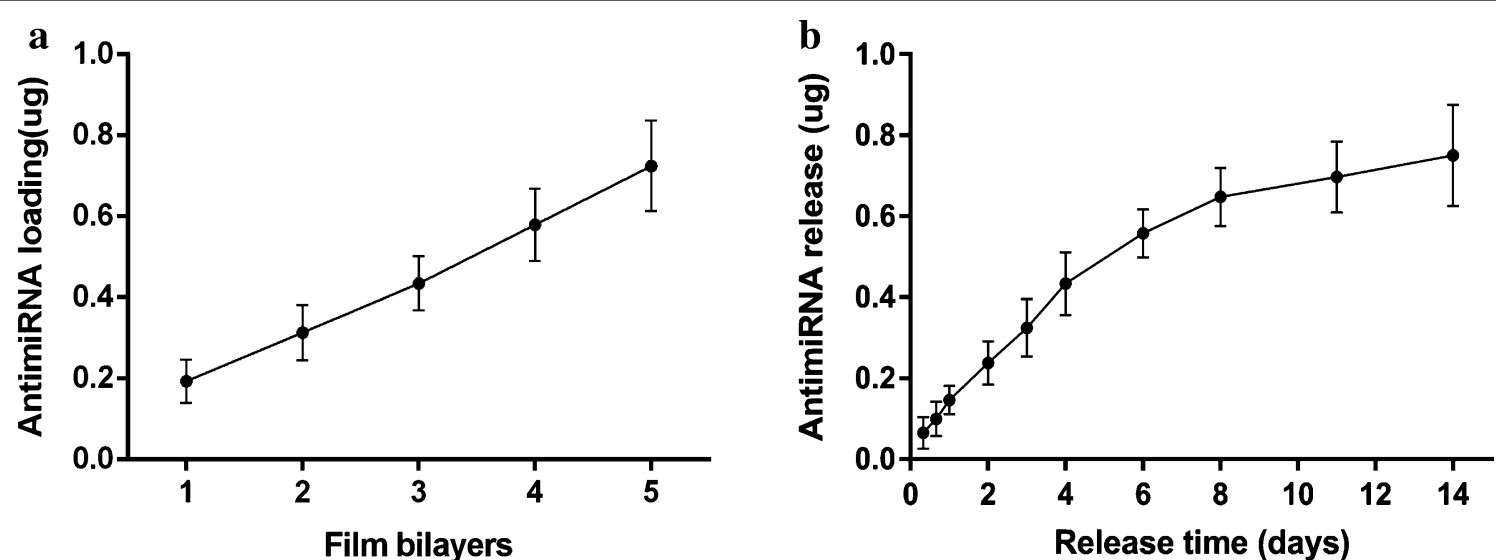

Fig. 4 Quantifcation of antimiR-138 on novel CS-antimiR-138/HA PEM-functionalized MAO surface. a Accumulated antimiR-138 loading amount with increased number of biofunctional layers, $\mathbf{b}$ accumulated antimiR-138 release profile from the coated surface 

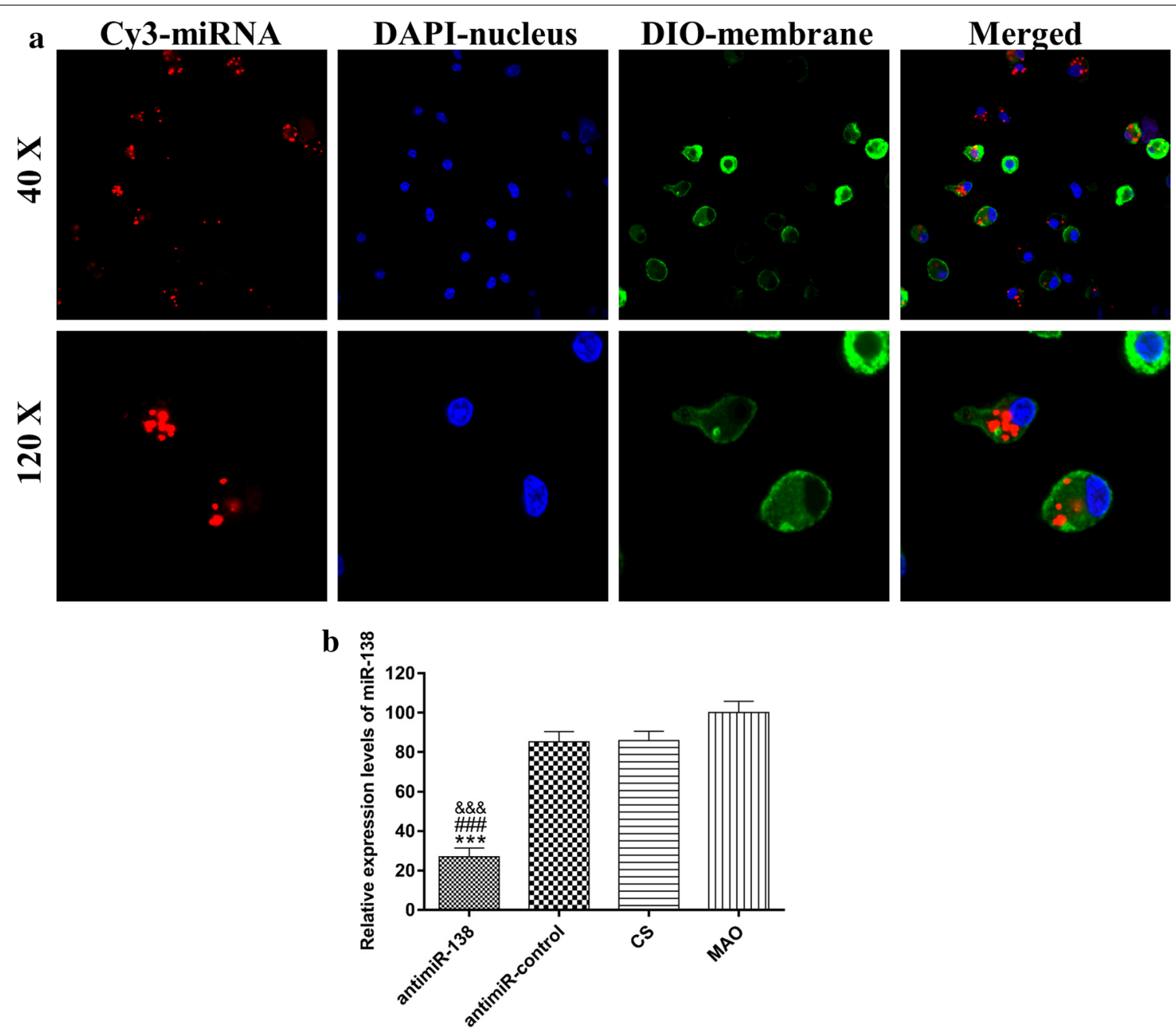

Fig. 5 a Fluorescence images of $40 \times$ (upper) and 120x (lower) showing the uptake of Cy3-labelled miRNAs (red) by cells after $24 \mathrm{~h}$ of culture on the CS-miRNA/HA PEM functionalized MAO surface: Cell nucleus was visualized using DAPI (blue) and membrane using DIO (green). $\mathbf{b}$ Downregulation of microRNA expression by 5 layers miRNA coating. ${ }^{* *} p<0.001$ vs the CS-antimiR-control/HA PEM-functionalized MAO surface; $\# \#$ \#\# $p .001$ vs the CS-coated MAO surface; $\$ \$ \$ p<0.001$ vs the naked MAO surface

in the optical images after 14 days of culture on all four surfaces, and the CS-antimiR-138/HA PEM-functionalized microporous Ti surface generated the most mineralized nodules. The quantitative results reveal that the CS-antimiR-138/HA PEM-functionalized microporous Ti surface induced the formation of approximately twice as many mineralized nodules than the three control groups did.

\section{In vivo osseointegration of the CS-antimiR-138/HA PEM-functionalized microporous Ti implant}

Micro-CT analysis was performed to evaluate new bone formation and osseointegration in vivo (Fig. 9). The reconstructed 3D stereoscopic images of the new bone formation (yellow) around the implants are shown in
Fig. 9a. The quantitative evaluation of the bone-volume ratio (BV/TV) and trabecular architecture are shown in Fig. 9b. The analysis of BV/TV, Tb.Th, Tb.N and Tb.Sp in the ROI revealed that the bone trabecula around the PT surface was the thinnest and sparsest. Compared to the three MAO control groups, the CS-antimiR-138/ HA PEM-functionalized microporous Ti surface induced noticeably more new bone formation (with BV/TV and Tb.N increased by approximately $40 \%$ and $\mathrm{Tb} . \mathrm{Sp}$ decreased by approximately half $(p<0.05))$.

To evaluate the tissue healing progress around the implants, we performed a histological analysis of the bone-to-implant interface by Van Gieson staining. New bone (red) was formed around the implants in all groups 4 weeks after implantation (Fig. 10a). Compared to the 

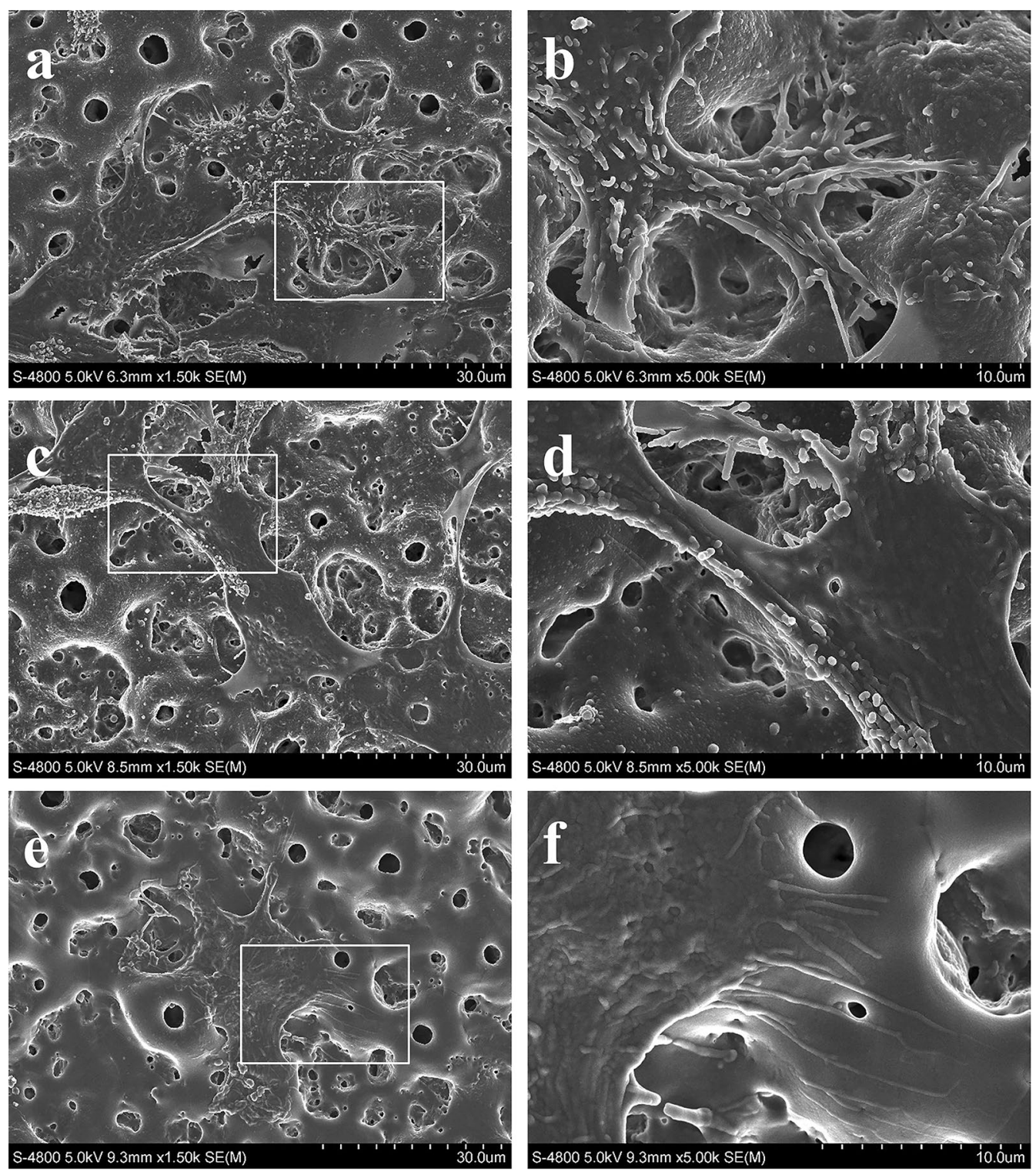

Fig. 6 FE-SEM pictures showing the cell morphology after $24 \mathrm{~h}$ of incubation on different samples: $\mathbf{a}$, $\mathbf{b}$ the CS-antimiR-138/HA PEM-functionalized MAO surface $(\mathbf{c}, \mathbf{d})$ the antimiR-control/HA PEM-functionalized MAO surface and $(\mathbf{e}, \mathbf{f})$ the naked MAO surface

PT implant with thin and discontinuous new bone formation, the CS-antimiR-control/HA PEM-functionalized microporous Ti implant, the CS-coated implant and the naked MAO implant induced more new bone formation. The CS-antimiR-138/HA PEM-functionalized microporous Ti implant generated the largest bone volume and the best bone continuity around the implant surface. Quantitative measurement of the BIC (Fig. 10b) shows a similar trend. The BIC increased from approximately $38 \%$ on the PT implant to approximately $85 \%$ on the CSantimiR-138/HA PEM-functionalized microporous $\mathrm{Ti}$ implant.
The cross-section of the bone-to-implant interface was inspected by FE-SEM (Fig. 11a). The distribution of elements including $\mathrm{C}, \mathrm{O}, \mathrm{P}, \mathrm{Ca}$, and $\mathrm{Ti}$ across the interface was analysed by EDX line scanning (Fig. 11b). The thickness of new bone with $\mathrm{Ca}$ and $\mathrm{P}$ was measured. The antimiR-138 PEM-functionalized microporous Ti surface induced the thickest new bone. The thickness of the new bone was approximately $18,25,27,27$, and $50 \mu \mathrm{m}$ for the PT implant, the naked MAO implant, the CS-coated MAO implant, the antimiR-control PEM-functionalized microporous Ti implant and the CS-antimiR-138/ 

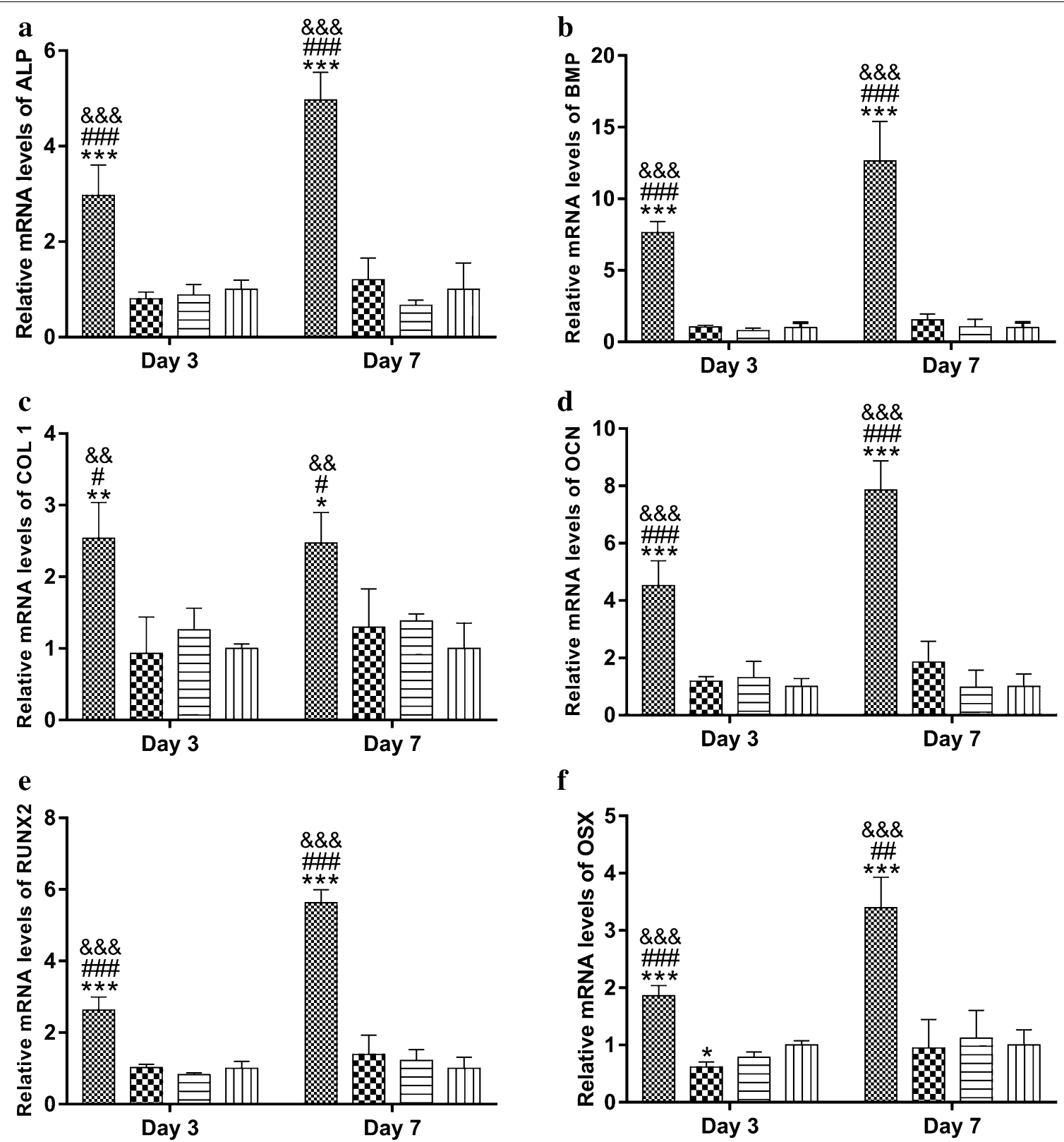

$\mathbf{f}$

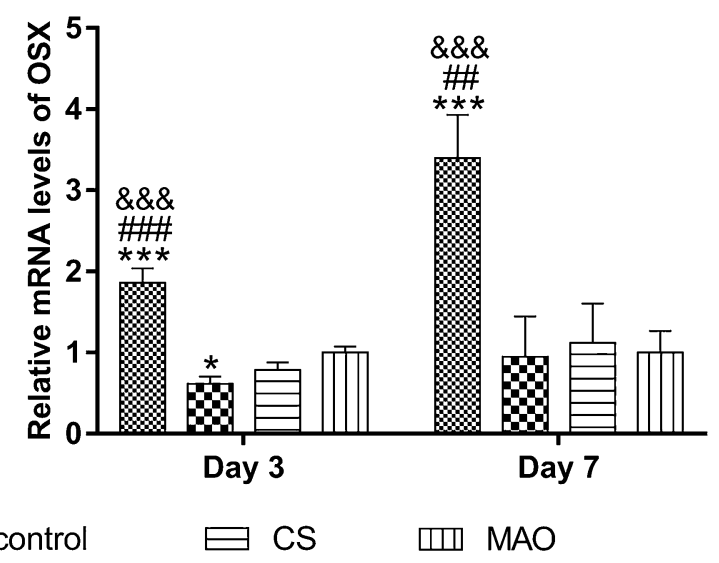

Fig. 7 Relative expression of $\mathbf{a} A L P, \mathbf{b} B M P, \mathbf{c}$ Col1, $\mathbf{d}$ OCN, e OSX and $\mathbf{f} \mathrm{RUNX} 2$ by MSCs cultured on different samples. After culturing in the growth medium for $24 \mathrm{~h}$, the medium was changed to osteogenic medium for further culture of 3 and 7 days. All values are normalized to GAPDH. ${ }^{*},{ }^{* *}$,

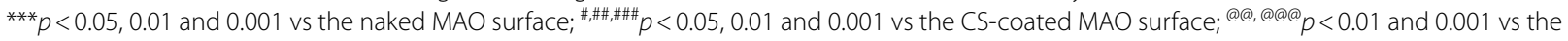
CS-antimiR-control/HA PEM-functionalized MAO surface

HA PEM-functionalized microporous $\mathrm{Ti}$ implant, respectively.

\section{Discussion}

The development of implants with quicker and tighter osseointegration is important to satisfy clinical requirements. Loading the implant surface with therapeutic oligonucleotides is a promising approach for accelerated tissue healing/integration [15, 42-44]. Song et al. created a siRNA biofunctionalized implant with a titanium nanotube array coating by cathodic electrodeposition of a CS-siRNA complex [45]. Previously, we conducted proof-of-concept studies to fabricate antimiR-138 lipoplex-functionalized Ti implants via lyophilization [22], 

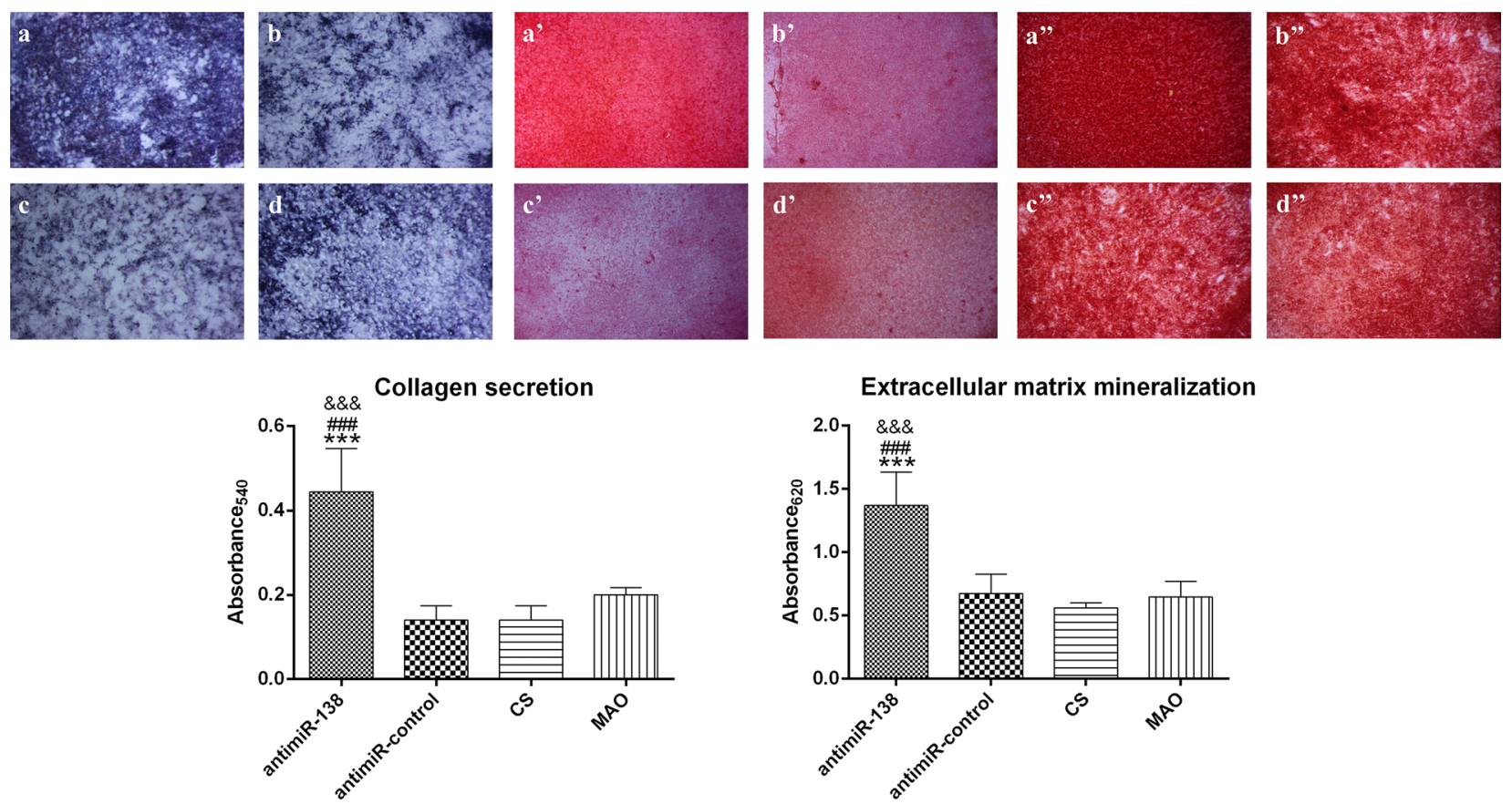

Extracellular matrix mineralization

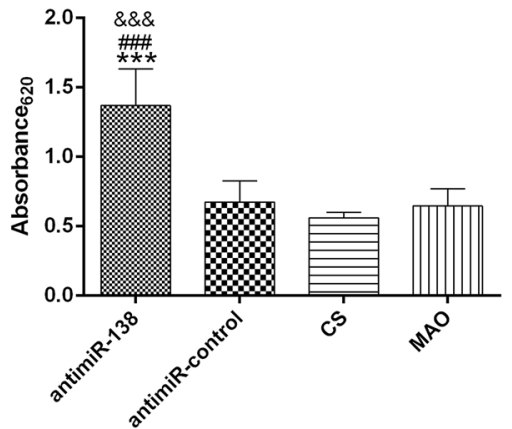

Fig. 8 The ALP product and collagen secretion after 7 days of culture as well as the ECM mineralization after 14 days of culture: (a, $\mathbf{a}^{\prime}$ and $\left.\mathbf{a}^{\prime \prime}\right)$ the CS-antimiR-138/HA PEM-functionalized MAO surface, $\left(\mathbf{b}, \mathbf{b}^{\prime}\right.$ and $\left.\mathbf{b}^{\prime \prime}\right)$ the CS-antimiR-control/HA PEM-functionalized MAO surface, (c, $\mathbf{c}^{\prime}$ and $\left.\mathbf{c}^{\prime \prime}\right)$ the CS-coated MAO surface and ( $\mathbf{d}, \mathbf{d}^{\prime}$ and $\mathbf{d}^{\prime \prime}$ ) the naked MAO surface. The bottom panel shows the semi-quantitative results. ${ }^{* *} p<0.001$ vs the naked MAO surface; ${ }^{\# \# ~} p<0.001$ vs CS-coated MAO surface; ${ }^{8 \& \&} p<0.001$ vs the CS-antimiR-control/HA PEM-functionalized MAO surface

which demonstrated the feasibility of the miRNA functionalization of Ti implants. However, a more biocompatible carrier instead of liposomes should be used to avoid cytotoxicity, and a sustainable release should be obtained by controlling the loading and delivery kinetics of antimiR-138.

In this study, multilayers of CS and HA fabricated by the LbL self-assembly technique were used to load miRNA on the Ti implant surface to realize a larger miRNA loading amount and a long-lasting release. This technique is based on the alternate adsorption of polyanions and polycations via electrostatic interactions on a charged substrate and has been widely used for the controlled release of biological molecules [46-48]. To firmly attach the LbL films to the MAO Ti surface, the silane reaction is utilized, as the rigid covalent bond provides a firm chemically crosslinked connection between the MAO surface and chitosan. UV irradiation produces abundant hydroxyl groups on the MAO surface, which can be reacted with the ethoxy groups of APTES [37]. The terminal amine group can then be reacted with the aldehyde groups of glutaraldehyde, which is commonly applied for crosslinking with biomolecules $[49,50]$. Then, chitosan can be covalently conjugated to the MAO Ti surface via the APTES-GA layer, which guaranteed the stability of the self-assembled multilayers on the $\mathrm{Ti}$ surface. Furthermore, due to its hydrophobicity and positive charge, chitosan was used as the superficial layer to promote protein adsorption and cell adhesion [51]. The FE-SEM images reveal that the MAO treatment generated a microporous structure topography with potential higher loading capacity [22]. The following silane reaction and PEM deposition formed a homogeneous surface coating without affecting the original MAO microtopography. Using laser scanning confocal microscopy, it was shown that the CS-miRNA complexes indeed entered deeply into the micropores and adhered to the inner sidewalls. The contact angle results again demonstrated that the self-assembled multilayers of chitosan and HA were successfully introduced onto the MAO Ti surface $[52,53]$.

The miRNA loading profile was analysed by fluorescence intensity measurement, which is one of the most sensitive methods for RNA quantification [54]. The cumulative loading amount of miRNA increased linearly with the number of layers. The in vitro miRNA release profile exhibited a phase of linear and fast release in the first 4 days, followed by a slower release phase. The observed long-lasting release kinetics can be attributed to the electrostatic interaction between the negatively and positively charged polymers in the PEM [30, 55, 56]. Furthermore, the MAO micropores can protect the 

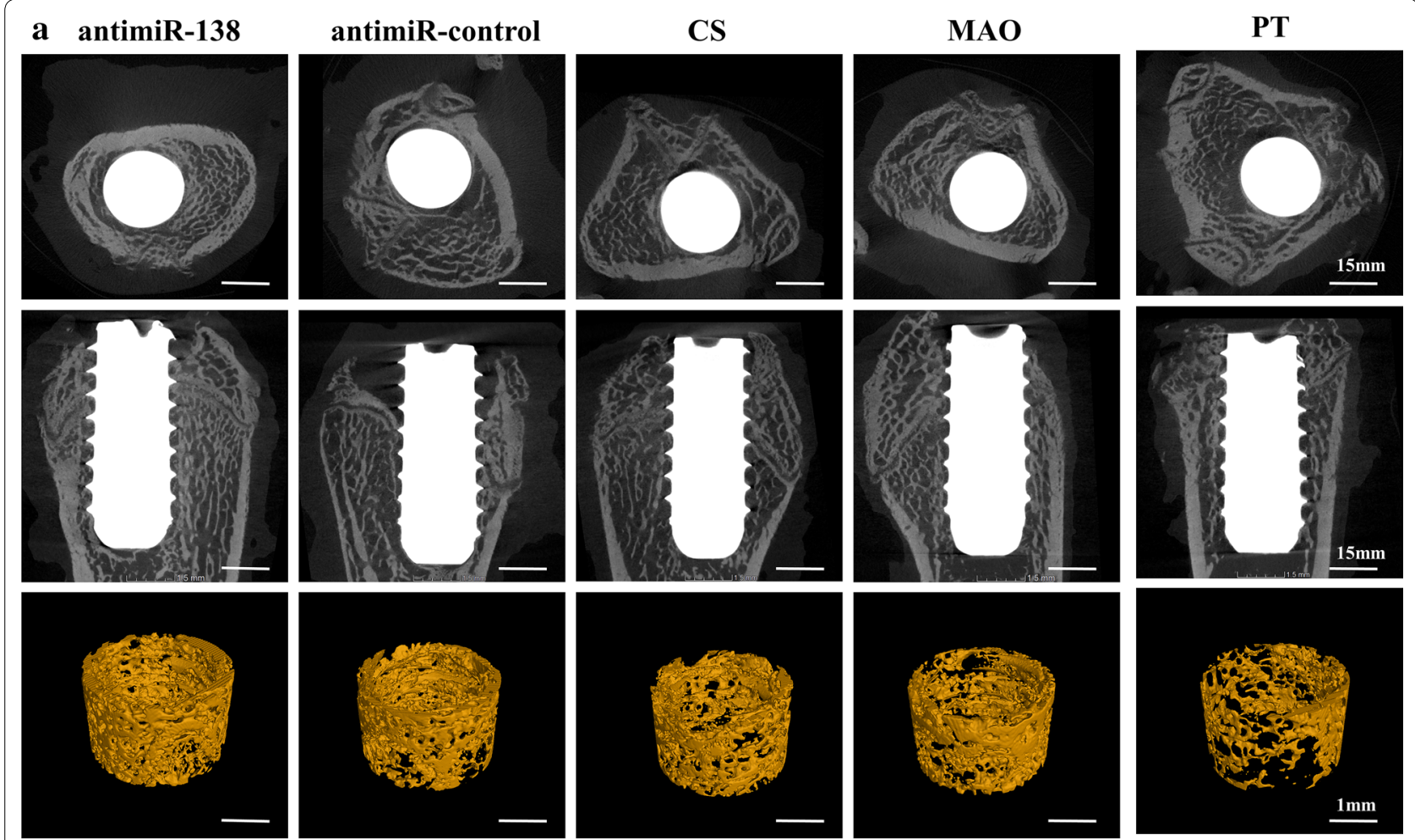

b
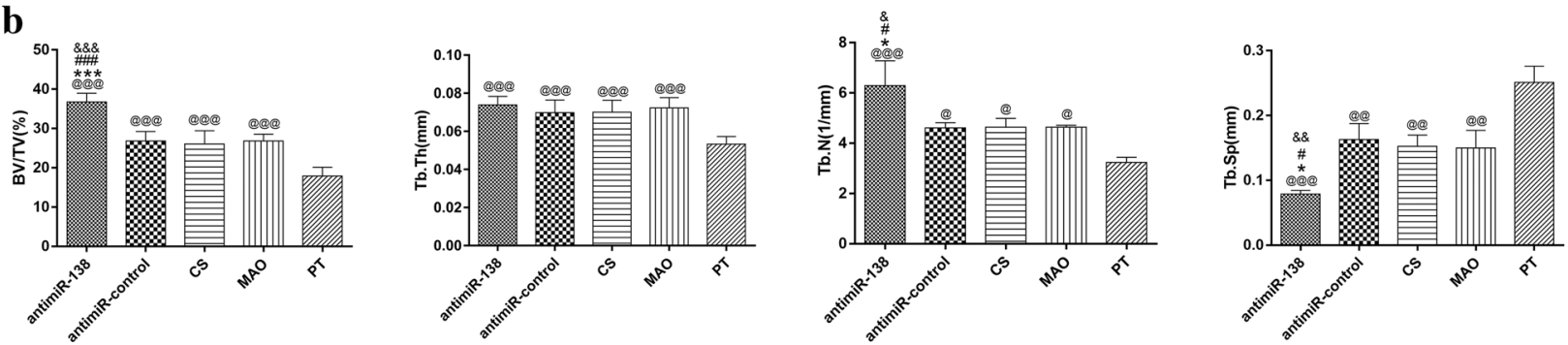

Fig. 9 a Transverse and vertical 2-D images and 3-D reconstructed views (ROI, $200 \mu \mathrm{m}$ from the implant surface) of the Micro-CT analysis to show the new bone formation around the Ti implants at 4 weeks. Scale bar: $15 \mathrm{~mm}$ on the 2-D images and $1 \mathrm{~mm}$ on the 3-D ones. b The bone volume per total volume (BV/TV), the mean trabecular thickness (Tb.Th), the mean trabecular number (Tb.N) and the mean trabecular separation (Tb.Sp)

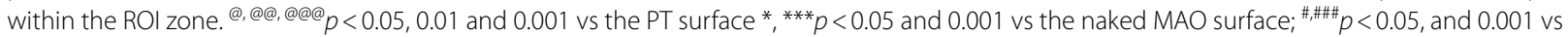
the CS-coated MAO surface; \&\&\&\&\&\& $p<0.05,0.01$ and 0.001 vs the CS-antimiR-control/HA PEM-functionalized MAO surface

nanoparticles from premature release due to an increased contact area and reduced shear force, contributing to the long-lasting release. Taken together, a long-lasting miRNA release of over 2 weeks was observed from the PEM-functionalized MAO Ti surface.

Then, the miRNA cellular uptake and the transfection efficiency of the CS-antimiR-138/HA PEM-functionalized microporous Ti surface were assessed. After cell plating on CS-antimiR-138/HA PEM-functionalized microporous Ti surfaces and transfection for $24 \mathrm{~h}$, abundant miRNAs were taken up by the cells, indicating a high transfection efficiency. This was further verified by miRNA-PCR. The intracellular target miRNA level was successfully downregulated by approximately $70 \%$. The high transfection efficiency was expected to be related to the microporous Ti surface and the miRNAs retained in the micropores, which leads to a localized concentration of miRNA. The direct cellular contact to this localized availability of nucleic acids results in improved transfection efficiency [57-59]. This effect is known as substrate-mediated gene delivery $[57,60]$. In addition, it has been reported that stability of CS/pDNA or CS/RNA complexes would not change in storage under $4{ }^{\circ} \mathrm{C}[61,62]$. Here, the CSantimiR-138/HA PEM-functionalized microporous Ti surface show consistent transfection efficiency over 

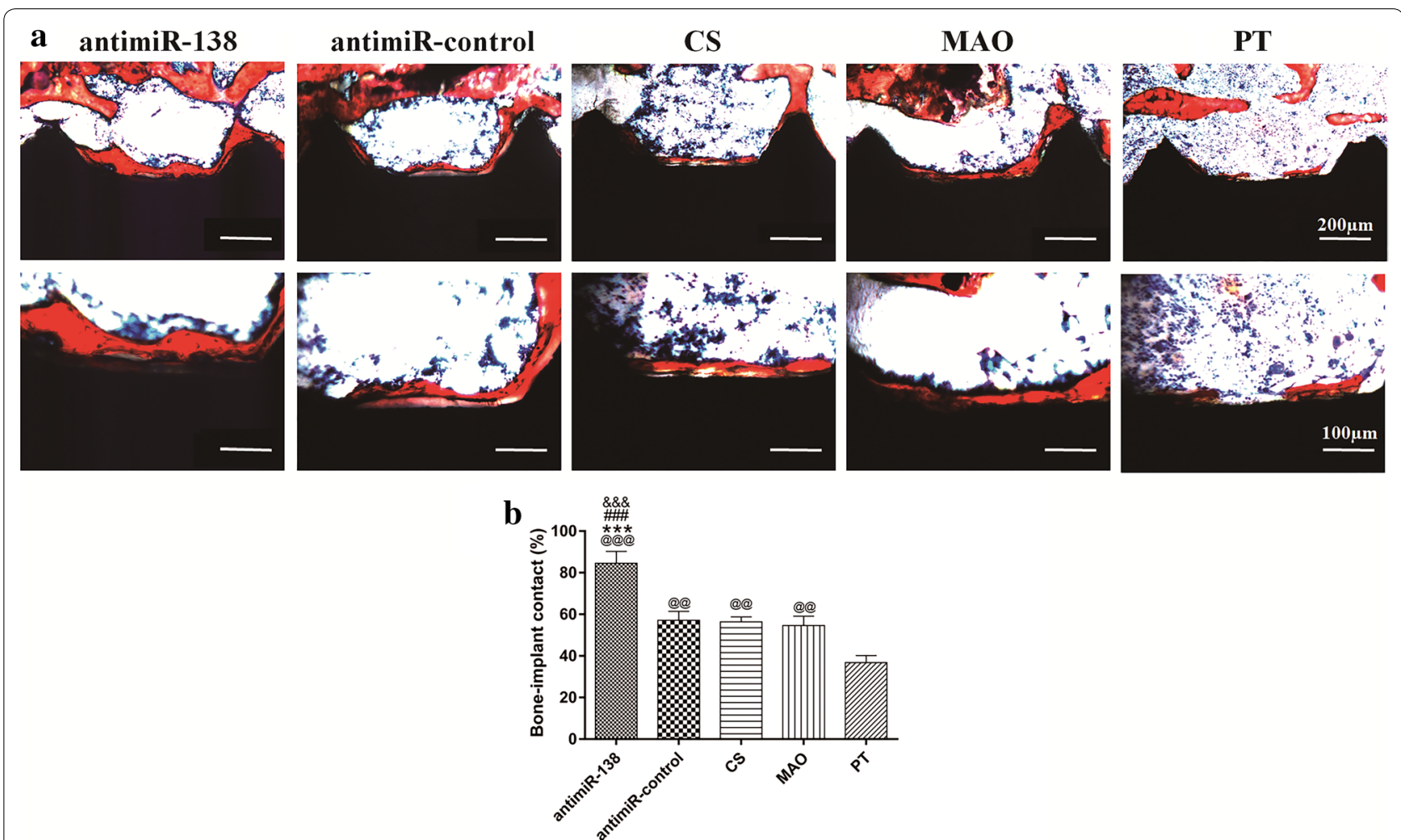

Fig. 10 a Histological images of undecalcified sections of new bone formation around the implants at 4 weeks by Van Gieson staining. The bone tissue was stained in red color. Scale bar: $200 \mu \mathrm{m}$ on the above and $100 \mu \mathrm{m}$ on the below. b Histomorphometric measurement of the BIC in the ROI. @@@@@ $p<0.01$ and 0.001 vs the PT surface ${ }^{* * *} p<0.001$ vs the naked MAO surface; ${ }^{\# \# \# ~} p<0.001$ vs the CS-coated MAO surface; ${ }^{* 8 \&} p<0.001$ vs CS-antimiR-control/HA PEM-functionalized MAO surface
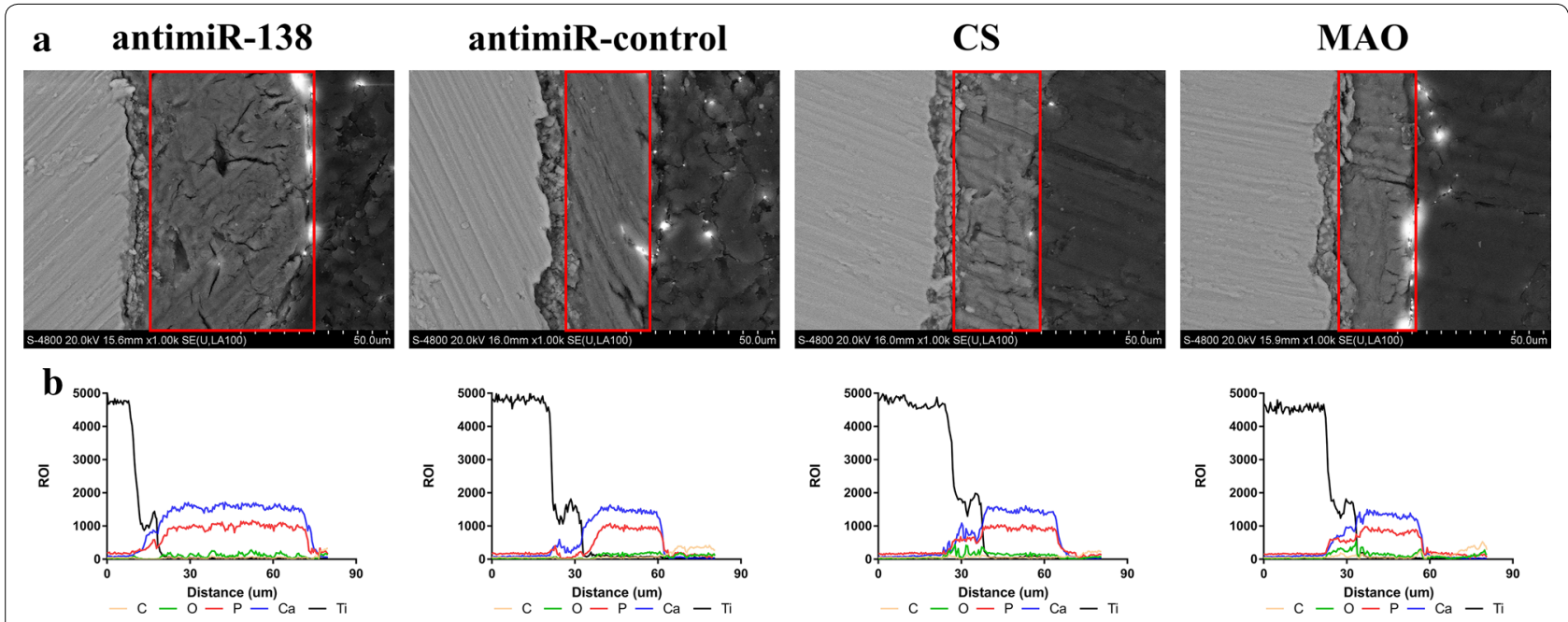

Fig. 11 a FE-SEM pictures showing the new bone on the bone-to-implant interface of different samples. $\mathbf{b}$ EDX line scanning of the elements in the direction perpendicular to the bone-to-implant interface. Red frame indicates the new bone area on the implant surface

14 days when stored at $4{ }^{\circ} \mathrm{C}$ (Additional file 1: Fig. S1 and $5 \mathrm{~b}$ ), indicating good storability for transportation and use.
High cytocompatibility without cytotoxicity or impairment of cell functions is an elementary requirement for the clinical application of biofunctionalized 
implants. Our data demonstrate that the CS-antimiR-138/HA PEM-functionalized microporous Ti surface satisfies this requirement (Additional file 1: Fig. S2). This is attributed to the biocompatibility of CS and HA and APTES/GA functional groups in the PEM. Nolte et al. found very low toxicity of HA/CS coatings [63]. Kuddannaya et al. found that poly(dimethylsiloxane) surfaces functionalized with APTES + GA + proteins showed enhanced MSC osteogenic induction with no cytotoxicity, which was related to the APTES/GA functional groups $[49,64]$. SU-8 surfaces functionalized with collagen type I or fibronectin via covalent conjugation through APTES + GA were biocompatible and used for long-term cell culture [65]. The cell attachment and spread influence the ensuing cell functions, and were observed by FE-SEM. The cells exhibited a very similar cell spreading behaviour with abundant cell lamellipodia stretching out on all surfaces, which further illustrates the high cytocompatibility of the CSantimiR-138/HA PEM-functionalized microporous $\mathrm{Ti}$ surface. The CS-antimiR-138/HA PEM-functionalized MAO Ti surface induced thicker filopodia, which were anchored in the micropores and may lead to a more rigid osseointegration. A more mature secretory cell phenotype was observed on the CS-antimiR-138/HA PEM-functionalized MAO Ti surface, which is likely due to the inhibition of the intracellular miR-138 function by antimiR-138 to the promote osteogenic differentiation of MSCs [49].

The use of a proper miRNA is pivotal for achieving satisfactory osteogenic activity for the PEM-functionalized implant surface. miR-138 is an important regulator in the development of the osteoblast phenotype $[18,22,66]$. It has been reported that miR-138 inhibits osteogenic differentiation of MSCs in vitro and the formation of bone in vivo. By inhibiting the focal adhesion kinase signalling pathway, antimiR-138 can promote the expression of osteoblast-specific genes, ALP activity, and ECM mineralization of MSCs, and enhance in vivo ectopic bone formation, suggesting that antimiR-138 is a good therapeutic molecule for enhancing osteogenesis [18]. Yan et al. also confirmed that antimiR-138 delivery increased the phosphorylation of ERK1/2, and subsequently increased the osteogenesis-related genes in antimiR-138-transfected MSCs [67]. Tsukamoto et al. found that the inhibition of miR-138 with an LNA-modified anti-miR-138 oligonucleotide enhanced the osteogenic differentiation of MSCs in vitro [68]. In addition, our previous data also demonstrated the efficiency of antimiR-138 in promoting osteogenic differentiation in vitro [22]. Therefore, the osteogenesis-inducing capacity of the antimiR-138-functionalized microporous coatings in vitro and in vivo was assessed. Excitingly, antimiR-138 resulted in enhanced osteogenic activity in vitro and excellent bone formation in vivo, which is consistent with the previous reports.

\section{Conclusions}

In this study, we developed a novel CS-antimiR-138/HA PEM-functionalized microporous Ti implant, which was prepared via silane-glutaraldehyde coupling to activate the MAO Ti surface and facilitate the chemical conjugation with biolayers composed of HA and chitosan using an LbL technique. The coating exhibited sustained release of CS-antimiR-138, which was taken up efficiently by the cells and caused significant knockdown of the target miR138 without inducing apparent cytotoxicity. Ti implants functionalized with antimiR-138 markedly enhanced the osteogenic differentiation of MSCs and the osseointegration in an in vivo rat model, thus being a very promising method to expedite the clinical implant osseointegration. This functionalization may thus represent a valuable tool for biomedical applications where a controlled, long-lasting delivery is needed and provide meaningful insights regarding miRNA loading into other biomaterials.

\section{Supplementary information}

Supplementary information accompanies this paper at https://doi. org/10.1186/s12951-020-00674-7.

Additional file 1: Figure S1. Storable stability of CS-antimiR-138/HA PEM-functionalized microporous Ti samples. After storage for different durations ( 7 and 14 days), the transfection efficiency was measured to assess the stability during storage. Figure S2. (a) Cell viability measured by CCK- 8 at $24 \mathrm{~h}$ after transfection and (b) LDH amount released by cells during the first $24 \mathrm{~h}$ after transfection.

\section{Acknowledgements}

Not applicable.

\section{Authors' contributions}

KMW and MYL performed in vitro study. KMW and NL performed in vivo study. MYL and NL analyzed the data. LZ and FHM offered help for the analysis of experimental data. KMW, MYL and NL drafted the main part of the manuscript, contributed equally to this work and should be considered as co-first authors. YMZ, LZZ, and ML designed and planned the experiments. KMW and LZZ wrote the manuscript. All authors provided input in the interpretation of results. All authors read and approved the final manuscript.

\section{Funding}

This study was granted by National Natural Science Foundation of China (Grant Nos. 31500776 and 31570954). Indigenous Innovation Program of Qingdao Municipal Science and Technology Commission (15-9-1-23-jch).

\section{Availability of data and materials}

All data generated or analyzed during this study are included in this published article.

\section{Ethics approval and consent to participate}

All animal experiments of this study were carried out at Experimental Animal Center, Fourth Military Medical University, and were approved by Experimental Animal Ethics Committee of the Fourth Military Medical University. 


\section{Competing interests}

The authors declare that they have no competing interests.

\section{Author details}

${ }^{1}$ Department of Stomatology, Navy 971st Hospital, No. 22 Minjiang Road, Qingdao 266071, China. ${ }^{2}$ Oral Research Center, Qingdao Municipal Hospital, Qingdao 266071, China. ${ }^{3}$ Third Department of Cadre's Ward, Navy 971st Hospital, Qingdao 266071, China. ${ }^{4}$ State Key Laboratory of Military Stomatology \& National Clinical Research Center for Oral Diseases \& Shaanxi Key Laboratory of Stomatology, Department of Prosthodontics, School of Stomatology, The Fourth Military Medical University, No. 145 West Changle Road, Xi'an 710032, China. ${ }^{5}$ State Key Laboratory of Military Stomatology \& National Clinical Research Center for Oral Diseases \& Shaanxi Engineering Research Center for Dental Materials and Advanced Manufacture, Department of Periodontology and Oral Medicine, School of Stomatology, The Fourth Military Medical University, No. 145 West Changle Road, Xi'an 710032, China.

Received: 14 May 2020 Accepted: 11 August 2020

Published online: 09 September 2020

\section{References}

1. Buser D, Sennerby L, De Bruyn H. Modern implant dentistry based on osseointegration: 50 years of progress, current trends and open questions. Periodontol. 2000;2017(73):7-21.

2. Civantos A, Martínez-Campos E, Ramos V, Elvira C, Gallardo A, Abarrateg A. Titanium coatings and surface modifications: toward clinically useful bioactive implants. ACS Biomater Sci Eng. 2017;3:1245-61.

3. Buser D, Broggini N, Wieland M, Schenk RK, Denzer AJ, Cochran DL, Hoffmann B, Lussi A, Steinemann SG. Enhanced bone apposition to a chemically modified SLA titanium surface. J Dent Res. 2004;83:529-33.

4. Park JW, Jang JH, Lee CS, Hanawa T. Osteoconductivity of hydrophilic microstructured titanium implants with phosphate ion chemistry. Acta Biomater. 2009:5:2311-21.

5. Iwata N, Nozaki K, Horiuchi N, Yamashita K, Tsutsumi Y, Miura H, Nagai A. Effects of controlled micro-/nanosurfaces on osteoblast proliferation. J Biomed Mater Res A. 2017;105:2589-96.

6. Rupp F, Gittens RA, Scheideler L, Marmur A, Boyan BD, Schwartz Z, Geis-Gerstorfer J. A review on the wettability of dental implant surfaces I: theoretical and experimental aspects. Acta Biomater. 2014;10:2894-906.

7. Yu Y, Jin G, Xue Y, Wang D, Liu X, Sun J. Multifunctions of dual Zn/Mg ion co-implanted titanium on osteogenesis, angiogenesis and bacteria inhibition for dental implants. Acta Biomater. 2017:49:590-603.

8. Lee S, Chang YY, Lee J, Madhurakkat Perikamana SK, Kim EM, Jung YH, Yun $\mathrm{JH}$, Shin $\mathrm{H}$. Surface engineering of titanium alloy using metal-polyphenol network coating with magnesium ions for improved osseointegration. Biomater Sci. 2020;8:3404-17.

9. Kilian KA, Bugarija B, Lahn BT, Mrksich M. Geometric cues for directing the differentiation of mesenchymal stem cells. Proc Natl Acad Sci USA. 2010;107:4872-7

10. Jurczak P, Witkowska J, Rodziewicz-Motowidlo S, Lach S. Proteins, peptides and peptidomimetics as active agents in implant surface functionalization. Adv Colloid Interface Sci. 2020;276:102083.

11. Heller M, Kumar VV, Pabst A, Brieger J, Al-Nawas B, Kämmerer PW. Osseous response on linear and cyclic RGD-peptides immobilized on titanium surfaces in vitro and in vivo. J Biomed Mater Res A. 2018;106:419-27.

12. Parfenova LV, Lukina ES, Galimshina ZR, Gil'fanova GU, Mukaeva VR, Farrakhov RG, Danilko KV, Dyakonov GS, Parfenov EV. Biocompatible organic coatings based on bisphosphonic acid RGD-derivatives for PEO-modified titanium implants. Molecules. 2020;25:229.

13. Kammerer PW, Pabst AM, Dau M, Staedt H, Al-Nawas B, Heller M. Immobilization of BMP-2, BMP-7 and alendronic acid on titanium surfaces: adhesion, proliferation and differentiation of bone marrow-derived stem cells. J Biomed Mater Res A. 2020;108:212-20.

14. Croes M, Akhavan B, Sharifahmadian O, Fan H, Mertens R, Tan RP, Chunara A, Fadzil AA, Wise SG, Kruyt MC, et al. A multifaceted biomimetic interface to improve the longevity of orthopedic implants. Acta Biomater. 2020;110:266-79

15. Song W, Yang C, Le Svend DQ, Zhang Y, Kjems J. Calcium-microRNA complex-functionalized nanotubular implant surface for highly efficient transfection and enhanced osteogenesis of mesenchymal stem cells. ACS Appl Mat Interfaces. 2018;10:7756-64.

16. Sakurai T, Yoshinari M, Toyama T, Hayakawa T, Ohkubo C. Effects of a multilayered DNA/protamine coating on titanium implants on bone responses. J Biomed Mater Res A. 2016;104:1500-9.

17. Carthew RW, Sontheimer EJ. Origins and mechanisms of miRNAs and siRNAs. Cell. 2009:136:642-55.

18. Eskildsen T, Taipaleenmaki H, Stenvang J, Abdallah BM, Ditzel N, Nossent AY, Bak M, Kauppinen S, Kassem M. MicroRNA-138 regulates osteogenic differentiation of human stromal (mesenchymal) stem cells in vivo. Proc Natl Acad Sci USA. 2011;108:6139-44.

19. Xu T, Luo Y, Wang J, Zhang N, Gu C, Li L, Qian D, Cai W, Fan J, Yin G. Exosomal miRNA-128-3p from mesenchymal stem cells of aged rats regulates osteogenesis and bone fracture healing by targeting Smad5. J Nanobiotechnol. 2020;18:47.

20. Zeng HC, Bae Y, Dawson BC, Chen Y, Bertin T, Munivez E, Campeau PM, Tao J, Chen R, Lee BH. MicroRNA miR-23a cluster promotes osteocyte differentiation by regulating TGF-beta signalling in osteoblasts. Nat Commun. 2017;8:15000

21. Elsafadi M, Manikandan M, Alajez NM, Hamam R, Dawud RA, Aldahmash A, lqbal Z, Alfayez M, Kassem M, Mahmood A. MicroRNA-4739 regulates osteogenic and adipocytic differentiation of immortalized human bone marrow stromal cells via targeting LRP3. Stem Cell Res. 2017;20:94-104.

22. Wu K, Song W, Zhao L, Liu M, Yan J, Andersen MO, Kjems J, Gao S, Zhang Y. MicroRNA functionalized microporous titanium oxide surface by lyophilization with enhanced osteogenic activity. ACS Appl Mat Interfaces. 2013:5:2733-44.

23. Abdelhamid HN, El-Bery HM, Metwally AA, Elshazly M, Hathout RM. Synthesis of CdS-modified chitosan quantum dots for the drug delivery of Sesamol. Carbohydr Polym. 2019;214:90-9.

24. Dowaidar M, Nasser Abdelhamid H, Hallbrink M, Langel U, Zou X. Chitosan enhances gene delivery of oligonucleotide complexes with magnetic nanoparticles-cell-penetrating peptide. J Biomater Appl. 2018;33:392-401.

25. Baghdan E, Pinnapireddy SR, Strehlow B, Engelhardt KH, Schafer J, Bakowsky U. Lipid coated chitosan-DNA nanoparticles for enhanced gene delivery. Int J Pharm. 2018;535:473-9.

26. Cupino TL, Watson BA, Cupino AC, Oda K, Ghamsary MG, Soriano S, Kirsch WM. Stability and bioactivity of chitosan as a transfection agent in primary human cell cultures: a case for chitosan-only controls. Carbohydr Polym. 2018;180:376-84.

27. Nguyen MA, Wyatt H, Susser L, Geoffrion M, Rasheed A, Duchez AC, Cottee ML, Afolayan E, Farah E, Kahiel Z, et al. Delivery of microRNAs by chitosan nanoparticles to functionally alter macrophage cholesterol efflux in vitro and in vivo. ACS Nano. 2019;13:6491-505

28. Jiang F, Yin F, Lin Y, Xia W, Zhou L, Pan C, Wang N, Shan H, Zhou Z, Yu X. The promotion of bone regeneration through CS/GP-CTH/antagomir-133a/b sustained release system. Nanomedicine. 2020;24:102116.

29. Decher G, Hong JD, Schmitt J, Decher G, Hong JD, Schmitt J. Buildup of ultrathin multilayer films by a self-assembly process: III. Consecutively alternating adsorption of anionic and cationic polyelectrolytes on charged surfaces. Thin Solid Films. 1992;210-211:831-5.

30. Del Mercato LL, Ferraro MM, Baldassarre F, Mancarella S, Greco V, Rinaldi R, Leporatti S. Biological applications of LbL multilayer capsules: from drug delivery to sensing. Adv Colloid Interface Sci. 2014;207:139-54.

31. Ding I, Shendi DM, Rolle MW, Peterson AM. Growth-factor-releasing polyelectrolyte multilayer films to control the cell culture environment. Langmuir. 2018;34:1178-89.

32. Shah NJ, Hyder MN, Quadir MA, Dorval Courchesne NM, Seeherman HJ, Nevins M, Spector M, Hammond PT. Adaptive growth factor delivery from a polyelectrolyte coating promotes synergistic bone tissue repair and reconstruction. Proc Natl Acad Sci USA. 2014;111:12847-52.

33. Cheng YC, Guo SL, Chung KD, Hu WW. Electrical field-assisted gene delivery from polyelectrolyte multilayers. Polymers. 2020;12:133.

34. Peng N, Yu H, Wang Z, Zhang Y, Deng K, Li J, Lu L, Zou T, Liu Y, Huang S. Dendrimer-grafted bioreducible polycation/DNA multilayered films with low cytotoxicity and high transfection ability. Mater Sci Eng C Mater Biol Appl. 2019;98:737-45.

35. Koenig O, Neumann B, Schlensak C, Wendel HP, Nolte A. Hyaluronic acid/ poly(ethylenimine) polyelectrolyte multilayer coatings for siRNA-mediated local gene silencing. PLoS ONE. 2019;14:e0212584 
36. Ghiorghita CA, Bucatariu F, Dragan ES. Influence of cross-linking in loading/ release applications of polyelectrolyte multilayer assemblies. A review. Mater Sci Eng C Mater Biol Appl. 2019;105:110050.

37. Han Y, Chen D, Sun J, Zhang Y, Xu K. UV-enhanced bioactivity and cell response of micro-arc oxidized titania coatings. Acta Biomater. 2008:4:1518-29.

38. Renoud P, Toury B, Benayoun S, Attik G, Grosgogeat B. Functionalization of titanium with chitosan via silanation: evaluation of biological and mechanical performances. PLoS ONE. 2012;7:e39367.

39. Norowski PA, Courtney HS, Babu J, Haggard WO, Bumgardner JD. Chitosan coatings deliver antimicrobials from titanium implants: a preliminary study. Implant Dent. 2011;20:56-67.

40. Lv H, Chen Z, Yang X, Cen L, Zhang X, Gao P. Layer-by-layer self-assembly of minocycline-loaded chitosan/alginate multilayer on titanium substrates to inhibit biofilm formation. J Dent. 2014;42:1464-72.

41. Dang Y, Zhang L, Song W, Chang B, Han T, Zhang Y, Zhao L. In vivo osseointegration of Ti implants with a strontium-containing nanotubular coating. Int J Nanomed. 2016;11:1003-11.

42. Zhang L, Zhou Q, Song W, Wu K, Zhang Y, Zhao Y. Dual-functionalized graphene oxide based siRNA delivery system for implant surface biomodification with enhanced osteogenesis. ACS Appl Mat Interfaces. 2017:9:34722-35.

43. Huang $Y$, Zheng Y, Xu Y, Li X, Zheng Y, Jia L, Li W. Titanium surfaces functionalized with siMIR31HG promote osteogenic differentiation of bone marrow mesenchymal stem cells. ACS Biomater Sci Eng. 2018;4:2986-93.

44. Xing H, Wang X, Xiao G, Zhao Z, Zou S, Li M, Richardson JJ, Tardy BL, Xie L, Komasa S, et al. Hierarchical assembly of nanostructured coating for siRNAbased dual therapy of bone regeneration and revascularization. Biomaterials. 2020;235:119784

45. Song W, Zhao L, Fang K, Chang B, Zhang Y. Biofunctionalization of titanium implant with chitosan/siRNA complex through loading-controllable and time-saving cathodic electrodeposition. J Mater Chem B. 2015;3:8567-76.

46. Yang Y, Zhu H, Wang J, Fang Q, Peng Z. Enzymatically disulfide-crosslinked chitosan/hyaluronic acid layer-by-layer self-assembled microcapsules for redox-responsive controlled release of protein. ACS Appl Mat Interfaces. 2018;10:33493-506.

47. Liu G, Li L, Huo D, Li Y, Wu Y, Zeng L, Cheng P, Xing M, Zeng W, Zhu C. A VEGF delivery system targeting Ml improves angiogenesis and cardiac function based on the tropism of MSCs and layer-by-layer self-assembly. Biomaterials. 2017:127:117-31.

48. I labal MH, Schroder A, Kerdjoudj H, Njel C, Senger B, Ball V, Meyer F, Boulmedais F. Effect of the buffer on the buildup and stability of tannic acid/ collagen multilayer films applied as antibacterial coatings. ACS Appl Mater Interfaces. 2020.

49. Kuddannaya S, Chuah YJ, Lee MH, Menon NV, Kang Y, Zhang Y. Surface chemical modification of poly(dimethylsiloxane) for the enhanced adhesion and proliferation of mesenchymal stem cells. ACS Appl Mat Interfaces. 2013;5:9777-84

50. Aydin EB, Aydin M, Sezginturk MK. Electrochemical immunosensor based on chitosan/conductive carbon black composite modified disposable ITO electrode: an analytical platform for p53 detection. Biosens Bioelectron. 2018;121:80-9.

51. Bumgardner JD, Wiser R, Elder SH, Jouett R, Yang Y, Ong JL. Contact angle, protein adsorption and osteoblast precursor cell attachment to chitosan coatings bonded to titanium. J Biomater Sci Polym Ed. 2003;14:1401-9.

52. Costa RR, Custodio CA, Arias FJ, Rodriguez-Cabello JC, Mano JF. Layer-bylayer assembly of chitosan and recombinant biopolymers into biomimetic coatings with multiple stimuli-responsive properties. Small. 2011;7:2640-9.

53. Song W, Song X, Yang C, Gao S, Klausen LH, Zhang Y, Dong M, Kjems J. Chitosan/siRNA functionalized titanium surface via a layer-by-layer approach for in vitro sustained gene silencing and osteogenic promotion. Int J Nanomedicine. 2015;10:2335-46.

54. Jones $\sqcup$, Yue ST, Cheung CY, Singer VL. RNA quantitation by fluorescencebased solution assay: riboGreen reagent characterization. Anal Biochem. 1998;265:368-74.

55. Luo J, Cao S, Chen X, Liu S, Tan H, Wu W, Li J. Super long-term glycemic control in diabetic rats by glucose-sensitive LbL films constructed of supramolecular insulin assembly. Biomaterials. 2012;33:8733-42.

56. Wohl BM, Engbersen JF. Responsive layer-by-layer materials for drug delivery. J Control Release. 2012;158:2-14.
57. Jewell CM, Lynn DM. Surface-Mediated Delivery of DNA: cationic polymers take charge. Curr Opin Colloid Interface Sci. 2008;13:395-402.

58. Lee EJ, Robinson TM, Tabor JJ, Mikos AG, Suh J. Reverse transduction can improve efficiency of AAV vectors in transduction-resistant cells. Biotechnol Bioeng. 2018;115:3042-9.

59. Li BC, Chang H, Ren KF, Ji J. Substrate-mediated delivery of gene complex nanoparticles via polydopamine coating for enhancing competitiveness of endothelial cells. Colloids Surf B Biointerfaces. 2016;147:172-9.

60. Holmes CA, Tabrizian M. Substrate-mediated gene delivery from glycolchitosan/hyaluronic acid polyelectrolyte multilayer films. ACS Appl Mat Interfaces. 2013:5:524-31.

61. Cifani N, Chronopoulou L, Pompili B, Di Martino A, Bordi F, Sennato S, Di Domenico EG, Palocci C, Ascenzioni F. Improved stability and efficacy of chitosan/pDNA complexes for gene delivery. Biotechnol Lett. 2015;37:557-65.

62. Katas H, Raja MA, Lam KL. Development of chitosan nanoparticles as a stable drug delivery system for protein/siRNA. Int J Biomater. 2013;2013:146320

63. Nolte A, Hossfeld S, Schroeppel B, Mueller A, Stoll D, Walker T, Wendel HP, Krastev R. Impact of polyelectrolytes and their corresponding multilayers to human primary endothelial cells. J Biomater Appl. 2013;28:84-99.

64. Chuah YJ, Kuddannaya S, Lee MH, Zhang Y, Kang Y. The effects of poly(dimethylsiloxane) surface silanization on the mesenchymal stem cell fate. Biomater Sci. 2015;3:383-90.

65. Xue P, Bao J, Chuah YJ, Menon NV, Zhang Y, Kang Y. Protein covalently conjugated SU-8 surface for the enhancement of mesenchymal stem cell adhesion and proliferation. Langmuir. 2014;30:3110-7.

66. Yan J, Chang B, Hu X, Cao C, Zhao L, Zhang Y. Titanium implant functionalized with antimiR-138 delivered cell sheet for enhanced peri-implant bone formation and vascularization. Mater Sci Eng C Mater Biol Appl. 2018:89:52-64.

67. Yan J, Zhang C, Zhao Y, Cao C, Wu K, Zhao L, Zhang Y. Non-viral oligonucleotide antimiR-138 delivery to mesenchymal stem cell sheets and the effect on osteogenesis. Biomaterials. 2014;35:7734-49.

68. Tsukamoto S, Løvendorf MB, Park J, Salem KZ, Reagan MR, Manier S, Zavidij O, Rahmat M, Huynh D, Takagi S, et al. Inhibition of microRNA-138 enhances bone formation in multiple myeloma bone marrow niche. Leukemia. 2018:32:1739-50.

\section{Publisher's Note}

Springer Nature remains neutral with regard to jurisdictional claims in published maps and institutional affiliations.

Ready to submit your research? Choose BMC and benefit from

- fast, convenient online submission

- thorough peer review by experienced researchers in your field

- rapid publication on acceptance

- support for research data, including large and complex data types

- gold Open Access which fosters wider collaboration and increased citations

- maximum visibility for your research: over $100 \mathrm{M}$ website views per year

At BMC, research is always in progress.

Learn more biomedcentral.com/submissions 